\title{
Satzstruktur und adverbiale Subordination. Eine Studie zum Deutschen und zum Italienischen*
}

\author{
Hardarik Blühdorn (Mannheim) und Miriam Ravetto (Vercelli)
}

\begin{abstract}
This paper investigates the syntactic behaviour of adverbial clauses in contemporary German and Italian. It focuses on three main questions: (i) How many degrees of syntactic integration of adverbial clauses are there to be distinguished by an adequate grammatical description of the two languages? (ii) Which linear and hierarchical positions in the structure of the matrix sentence can be occupied by adverbial clauses? (iii) Which is the empirical distribution of adverbial clauses introduced by the conjunctions als, während, wenn, obwohl and weil in German, as well as quando, mentre, se, sebbene and perché in Italian?

Responding to question (i), a distinction is drawn between strongly integrated, weakly integrated and syntactically disintegrated adverbial clauses. There are further degrees on the gradient of syntactic integration, which are not examined in this paper. Responding to question (ii), eight classes of structural positions in the matrix sentence are identified that can be occupied by adverbial clauses. Five of them are positions of syntactic integration, three are positions of disintegration. Responding to question (iii), the distribution of the ten classes of adverbial clauses is described on the basis of a corpus of internet data. Strongly integrated, weakly integrated and disintegrated adverbial clauses show clearly different distributions within the structure of the matrix sentence. Also the semantic classes of adverbial clauses (temporal, adversative, conditional, concessive, causal) are distributed differently.
\end{abstract}

\section{$1 \quad$ Einleitung}

In der traditionellen Grammatikschreibung wird zwischen Koordination und Subordination als Grundverfahren der Satzverknüpfung unterschieden. Als Koordination wird eine Verknüpfung syntaktisch gleichberechtigter Ausdrücke bezeichnet, als Subordination eine Verknüpfung von Ausdrücken, die in einem hierarchischen Gefälle zueinander stehen. Für beide Verfahren ist das Vorkommen eines konjunktionalen Verknüpfungsausdrucks (Konnek-

\footnotetext{
* Wir danken unseren KollegInnen Marina Foschi Albert, Patrizio Malloggi und Bernd Wiese sowie zwei anonymen LO-GutachterInnen für Diskussion und wertvolle Hinweise. Miriam Ravetto dankt der Alexandervon-Humboldt-Stiftung für die Förderung ihres Forschungsaufenthalts am Institut für Deutsche Sprache in Mannheim (April-Juni 2013; Vorgangsnummer ITA/1148681 STP). Beide Autoren haben gemeinsam das Korpus zusammengestellt, die Daten untersucht, die verwendete Fachliteratur gelesen und die Untersuchung zum Deutschen und Italienischen durchgeführt. Hardarik Blühdorn hat die Abschnitte 1, 2 und 3 geschrieben; Miriam Ravetto hat die Abschnitte 4 und 5 verfasst.
} 
tors) typisch, der Hinweise darauf gibt, wie die Verknüpfungsrelation semantisch interpretiert werden soll (vgl. Helbig/Buscha 2001: 561-613).

In jüngeren Arbeiten ist öfter vorgeschlagen worden, das Feld der Satzverknüpfungen als Kontinuum zu konzeptualisieren, in dem mehr als zwei syntaktische Verfahren unterschieden werden können (vgl. etwa Kortmann 1996: 56-58; Johannessen 1998: 237-251; Cristofaro 2003: 22-25). Der eine Pol einer solchen Skala ist dadurch gekennzeichnet, dass die verknüpften Ausdrücke strukturell gleichrangig nebeneinanderstehen. Ihm entspricht nicht die klassische Koordination durch einen Konjunktor im Sinne von Pasch et al. (2003: 453-481), sondern die asyndetische Reihung: Zwei syntaktisch voneinander unabhängige Ausdrücke folgen ohne Bindeglied aufeinander. In welcher semantischen Relation sie zueinander stehen, muss der Interpret selbständig erschließen (vgl. Helbig/Buscha 2001: 561).

Die Koordination durch einen Konjunktor zeigt bereits eine strukturelle Asymmetrie zwischen den verknüpften Ausdrücken: Der Konjunktor ist mit einem seiner Konjunkte syntaktisch enger verbunden als mit dem anderen - wie auch immer man diese Asymmetrie im Detail explizieren mag (vgl. u. a. Johannessen 1998: 108-174; Haspelmath 2004: 5-10; Carston/Blakemore 2005: 354; Blühdorn 2008b: 62f;; Blühdorn 2008c: 4-7; Blühdorn 2012a: 199-205). Den anderen Pol der Skala bilden maximal asymmetrische Verknüpfungen, in denen ein Ausdruck vollständig als Konstituente in den anderen integriert und ihm in diesem Sinne syntaktisch subordiniert ist (vgl. Zifonun et al. 1997: 2235-2358). Zwischen Koordination und vollständiger Subordination liegen Grade unvollständiger Subordination (vgl. ebd.: 2250-2253).

Der vorliegende Aufsatz untersucht unterschiedliche Grade der syntaktischen Subordination adverbialer Nebensätze im Deutschen und Italienischen. Er soll zeigen, dass und auf welche Weise subordinierende Satzverknüpfungen in beiden Sprachen mit einheitlichen Darstellungswerkzeugen beschrieben und verglichen werden können. Wir beschränken die Untersuchung auf Satzverknüpfungen, in denen einer der Teilsätze die Funktion eines adverbialen Adjunkts in Bezug auf den anderen (seinen Wirt) übernimmt. Adverbiale Adjunkte sind erweiternde Konstituenten, die keine syntaktischen Leerstellen sättigen und die syntaktische Kategorie ihres Wirtes nicht verändern (vgl. Sternefeld 2008/2009: 719-737; Blühdorn/Lohnstein 2012: 188-193).

In Kapitel 2 diskutieren wir Vorschläge aus der aktuellen Fachliteratur zur Unterscheidung von Subordinationsgraden und zum strukturellen Status von Adverbialia. In Unterkapitel 3.1 stellen wir ein aus der generativen Grammatik stammendes Strukturmodell für deutsche und italienische Sätze vor, mit dem deren linearer und hierarchischer Aufbau auf einheitliche Weise beschrieben werden kann. In Unterkapitel 3.2 unterscheiden wir neun Positionen in der linearen Satzstruktur, die für den deskriptiven Teil der Untersuchung benötigt werden. In Kapitel 4 gehen wir anhand empirischer Daten im Detail auf eine Auswahl von zehn Konjunktionen ein: fünf aus dem Deutschen - als, während, wenn, obwohl und weil - und fünf semantisch partiell äquivalenten aus dem Italienischen - quando, mentre, se, sebbene und perché. Kapitel 5 fasst die Ergebnisse zusammen. 
Die angeführten Beispiele stammen überwiegend aus dem Internet. ${ }^{1}$ Mit der Suchmaschine Google haben wir für jeden untersuchten Konnektor ein Korpus von 100 bis 200 Belegen zusammengestellt, das die Bandbreite seiner Verwendungsmöglichkeiten dokumentiert. Um die Aufmerksamkeit auf das Wesentliche zu lenken, haben wir manche Belege behutsam gekürzt, orthographisch korrigiert oder anderweitig bearbeitet, aber stets ohne diejenigen grammatischen Eigenschaften anzutasten, die zur Diskussion stehen. Ein kleinerer Teil der Beispiele entstammt der Fachliteratur. Einige wenige, etwa bei der Anwendung oder Illustration von Testverfahren, haben wir selbst gebildet, jedoch stets mit Blick auf unsere Korpusdaten, um Artefakte zu vermeiden. Beispiele, die uns zweifelhaft erschienen, haben wir nur nach einer positiven Beurteilung durch Informanten aufgenommen. Um Platz zu sparen, wird die Herkunft der Belege nicht einzeln dokumentiert, da dies ihre Aussagekraft unseres Erachtens nicht erhöhen würde. Für die meisten dargestellten Erscheinungen lassen sich leicht weitere Belege im Internet finden. Wo dies nicht der Fall ist, weisen wir ausdrücklich darauf hin.

\section{$2 \quad$ Adverbiale Satzverknüpfungen}

In der einschlägigen Literatur sind unterschiedliche Vorschläge dazu gemacht worden, wie viele Integrationsgrade für adverbiale Satzverknüpfungen unterschieden werden müssen.

\subsection{Zentrale vs. periphere Adverbialsätze}

Haegeman (2003; 2004; 2006; 2012: 149-194) unterscheidet zwischen zentralen und peripheren Adverbialsätzen. Sie nimmt an, dass zentrale Adverbialsätze an die VP oder IP, periphere an die CP adjungiert sind, bzw. dass zentrale Adverbialsätze mit dem Matrixsatz vereinigt werden "before IP is completed", wohingegen periphere Adverbialsätze mit dem Matrixsatz vereinigt werden, "after (...) CP has been completed" (2002: 71; 2003: 326). ${ }^{2}$ Diese konkreten Strukturhypothesen macht sie allerdings nicht hauptsächlich mit syntaktischen Argumenten plausibel. Die Tests, mit denen sie zwischen den beiden Klassen von Adverbialsätzen unterscheidet (2002: 63-71), sind überwiegend semantischer Art.

Zentrale und periphere Adverbialsätze haben nach Haegemans Darstellung (2004: 61) unterschiedliche semantische Funktion. Zentrale Adverbialsätze dienen dazu, den im Matrixsatz beschriebenen Sachverhalt zu "strukturieren", d.h. ihn in eine zeitliche Beziehung zu anderen Sachverhalten zu setzen. Periphere Adverbialsätze haben demgegenüber die Aufgabe, den Diskurs zu "strukturieren", d. h. die Aussage bzw. den Sprechakt des Matrixsatzes in Beziehung zum Interaktionskontext zu setzen (vgl. Fabricius-Hansen 1992: 467-468; Pittner 1999: 320; Reich et al. 2009: 11; Axel-Tober 2012: 13f.). Zur Veranschaulichung zwei Beispiele aus dem Deutschen und zwei aus dem Italienischen:

(1) Wenn ein Kind verstanden und toleriert wird, lernt es, geduldig zu sein.

(2) Sie haben auch spezielle Angebote für Ski-Anfänger, wo du schon alles inklusive hast (Skipass, Unterkunft), wenn ich mich richtig erinnere.

\footnotetext{
1 Vgl. dazu auch den Nachsatz zum Verhältnis zwischen Grammatikfoschung und Sprachdaten am Ende des Aufsatzes.

${ }^{2} \mathrm{Zu}$ den Symbolen VP, IP und CP siehe Unterkapitel 3.1.
} 
(3) Se non stai fermo ti fai male.

Wenn du nicht still hältst, tust du dir weh. ${ }^{3}$

(4) Se non mi sbaglio, ci siamo già conosciuti.

Wenn ich mich nicht irre, haben wir uns schon kennengelernt.

Die wenn- bzw. se-Adverbialsätze in (1) und (3) beschreiben Sachverhalte, deren (Nicht-)Eintreten das Eintreten der im Matrixsatz beschriebenen Sachverhalte begünstigt. Solche Adverbialsätze sind im Sinne Haegemans zentral. Die Adverbialsätze in (2) und (4) beschreiben epistemische Bedingungen für das Zutreffen der mit den Matrixsätzen gemachten Aussagen (vgl. dazu Blühdorn 2008a). Solche Adverbialsätze sind peripher.

Zentrale und periphere Adverbialsätze unterscheiden sich hinsichtlich zahlreicher weiterer semantischer Kriterien. Unter anderem liegen zentrale Adverbialsätze, wenn der Matrixsatz einen Negations- oder Fokuspartikeloperator enthält, häufig in dessen Skopus. Dagegen können periphere Adverbialsätze vom Skopus eines solchen Operators im Matrixsatz nicht erfasst werden (vgl. Haegeman 2004: 64-68; Axel-Tober 2012: 9-10; Blühdorn 2012b: 259-260). Zwei Beispiele aus dem Deutschen mit Negation, zwei aus dem Italienischen mit Fokuspartikeln:

(5) Menschen kaufen Gold nicht, weil sie es brauchen, sondern weil sie es wollen.

(6) Pietro rinuncia solo all'amore perché non lo ha mai avuto.

Peter verzichtet nur auf die Liebe, weil er sie nie gehabt hat.

(7) Die Idee war nicht gut, wenn du mich fragst.

(8) Lucia sa addirittura il russo, se ben mi ricordo.

Lucia kann sogar Russisch, wenn ich mich richtig erinnere.

In (5) nimmt der Negationsoperator nicht Skopus über den weil-Satz. (6) kann - bei geeigneter Intonation - so gelesen werden, dass die Fokuspartikel solo Skopus über den perché-Satz nimmt. Adverbialsätze wie die in (5) und (6) sind zentral. In (7) und (8) können der wennbzw. der se-Satz nicht vom Skopus des Negations- bzw. Fokuspartikeloperators erfasst werden. Die Adverbialsätze sind peripher.

Haegeman (2003: 323f.) zufolge unterscheiden sich zentrale und periphere Adverbialsätze auch in ihrem Bindungsverhalten. Eine Variable in einem zentralen Adverbialsatz kann durch einen quantifizierenden Ausdruck im Matrixsatz gebunden werden. Mit Variablen in einem peripheren Adverbialsatz ist das nicht möglich:

(9) Otto darf gerne zu spät kommen, zumal er ja keine Uhr hat.

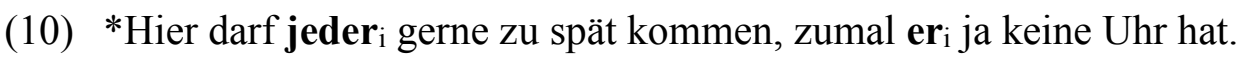

Zumal-Sätze können dem Matrixsatz nur nachgestellt werden (vgl. Pasch et al. 2003: 360). Sie können nicht im Skopus von Negationsoperatoren oder Fokuspartikeln im Matrixsatz liegen. Somit scheinen sie peripher zu sein. In (9) erlaubt das Pronomen er im zumal-Satz zwei Lesarten: Es kann koreferent mit dem Subjekt des Matrixsatzes (Otto) sein oder eventuell

\footnotetext{
${ }^{3} \mathrm{Zu}$ den italienischen Beispielen geben wir deutsche Übersetzungen in Kursivschrift, bei Bedarf wörtlich, um syntaktische Strukturen zu verdeutlichen.
} 
auch auf eine andere, im Kontext identifizierbare Person referieren. In jedem Fall muss es referentiell gelesen werden. Eine Lesart als gebundene Variable scheidet aus. In Beispiel (10) kann er eventuell referentiell in Bezug auf eine im Kontext identifizierbare Person gelesen werden. Eine Lesart als Variable, die durch den Quantifikator jeder gebunden wäre, kommt nicht in Betracht.

Deutlich weniger inakzeptabel als (10) ist allerdings (11). Nach Haegeman (2004: 61f.) sind auch adversative Adverbialsätze peripher. In (11) kann aber eine Lesart, in der er eine durch jeder gebundene Variable ist, keineswegs ausgeschlossen werden:

(11) Jeder deutsche Beamte ${ }_{i}$ kommt montags zu spät, während $\mathbf{e r}_{i}$ freitags zu früh geht.

Solche Beispiele deuten darauf hin, dass innerhalb der peripheren Adverbialsätze weitere Unterscheidungen notwendig sind.

Ein Beispielpaar mit unterschiedlichen Bindungsverhältnissen aus dem Italienischen:

(12) *Nessuno i può ascoltare le telefonate di nascosto, tanto più che $\boldsymbol{\emptyset}_{\mathrm{i}}$ ha solo il suo $\mathrm{i}_{\mathrm{i}}$ telefono personale.

*Niemand $d_{i}$ kann Telefongespräche heimlich mithören, zumal er ${ }_{i}$ nur sein $_{i}$ privates Telefon hat.

(13) Nessuno i può ascoltare le telefonate di nascosto, se $\boldsymbol{\emptyset}_{\mathrm{i}}$ ha solo il suo telefono personale.

Niemand ${ }_{i}$ kann Telefongespräche heimlich mithören, wenn er ${ }_{i}$ nur sein s $_{i}$ privates Telefon hat.

Tanto più che-Sätze wie in (12) sind ebenso wie zumal-Sätze immer nachgestellt und erfüllen die Kriterien für peripheren Status. In (12) kann das leere Subjekt im Adverbialsatz keine durch nessuno gebundene Variable sein. Wird der Adverbialsatz mit se angeschlossen wie in (13), ist eine entsprechende Bindung möglich. Dann ist der Adverbialsatz zentral.

Haegeman (2006: 1651; 2012: 182-194) stellt die These auf, zentrale Adverbialsätze hätten im Unterschied zu peripheren eine reduzierte CP. Dadurch erkläre sich ihr besonderes syntaktisch-semantisches Verhalten. Diese These übernehmen wir hier nicht. Auf eine Diskussion müssen wir aus Platzgründen verzichten.

Auch Frey (2011: 46-61) unterscheidet zwischen zentralen und peripheren Adverbialsätzen und weist auf weitere Unterschiede in ihrem grammatischen Verhalten hin. Zentrale Adverbialsätze sind seiner Darstellung nach in der Regel erfragbar, periphere nicht:

(14) Litigo con mio marito perché non voglio cani in casa. Ich streite mit meinem Mann, weil ich keine Hunde im Haus will.

(14a) Perché litighi con tuo marito? Perché non voglio cani in casa. Warum streitest du mit deinem Mann? Weil ich keine Hunde im Haus will.

(15) Weil es mir gerade einfällt: Die PC-Version von Minecraft wurde von der USK noch nicht eingestuft.

$(15 a) \neq$ Warum wurde die PC-Version von Minecraft von der USK noch nicht eingestuft? Weil es mir gerade einfällt. 
Im Gegensatz zu zentralen können periphere Adverbialsätze Abtönungs- bzw. Modalpartikeln enthalten (vgl. Coniglio 2011: 202; Frey 2011: 55-61):

(16) Als sie weinte, bekam sie Schläge.

(16a) *Als sie ja weinte, bekam sie Schläge.

(17) Un tempo si parlava molto di rock italiano al femminile, mentre ora semmai si parla soltanto di pop.

Früher war oft von weiblichem italienischem Rock die Rede, während man heute wenn überhaupt nur von Pop spricht.

In (16a) ist die Modalpartikel ja in dem temporalen als-Satz ungrammatisch. Der Nebensatz ist zentral. In dem peripheren zumal-Satz in (9) spricht nichts gegen die Modalpartikel. Ob es im Italienischen im gleichen Sinne wie im Deutschen Modalpartikeln gibt, ist umstritten (vgl. Coniglio 2011: 27-34). Ein möglicher Kandidat ist nach Buzzo Margari (2004: 256) semmai. In Beispiel (17) kann diese Partikel im Adverbialsatz stehen. Die Verknüpfung muss hier adversativ gelesen werden (vgl. Blühdorn/Ravetto 2012: 56f.). Adversative mentre-Sätze sind im Sinne Haegemans peripher (dazu ausführlicher unten Abschnitt 4.2).

Im geschriebenen Italienisch werden periphere Adverbialsätze im Gegensatz zu zentralen typischerweise durch Interpunktionszeichen wie Kommata oder Gedankenstriche vom Matrixsatz abgegrenzt, ähnlich wie im Englischen (vgl. Haegeman 2004: 68f.). Im Deutschen werden Nebensätze aller Art in Interpunktionszeichen eingeschlossen. Hier kann dieses Unterscheidungskriterium nicht herangezogen werden.

\subsection{Integrierte vs. desintegrierte abhängige Sätze}

Holler (2008) unterscheidet zwischen integrierten und desintegrierten abhängigen Sätzen. Zu den integrierten abhängigen Sätzen rechnet sie kanonische Nebensätze wie Subjekt-, Objektund Adverbialsätze mit Endstellung des Finitums; zu den desintegrierten rechnet sie sogenannte freie dass-Sätze wie in (18), weiterführende w-Relativsätze wie in (19), weil-Sätze mit Zweitstellung des Finitums (weil-V2-Sätze) wie in (20) und einige andere Satzarten:

(18) Max muss größenwahnsinnig sein, dass er sich jetzt den dritten Ferrari kauft.

(19) Keine Tür war verschlossen, was mich erstaunte.

(20) Ich denke, es gibt Leben auf dem Mars, weil man hat dort ja Eis gefunden.

Weil-V2-Sätze verhalten sich syntaktisch wie denn-Sätze. Nach Uhmann (1998) sind sie nicht als abhängig, sondern als koordiniert zu betrachten. Freie dass-Sätze und weiterführende wRelativsätze haben mehr Eigenschaften, die für syntaktische Abhängigkeit sprechen.

Holler zeigt, dass desintegrierte abhängige Sätze sich in Bezug auf Skopuseffekte, Variablenbindung und Korrelatfähigkeit von integrierten abhängigen Sätzen unterscheiden, also hinsichtlich der gleichen semantischen Kriterien, die auch im Zusammenhang mit der Unterscheidung zwischen zentralen und peripheren Nebensätzen angeführt worden sind (vgl. dazu auch Reis 1997). Daneben verwendet sie weitere Kriterien, die für die Unterscheidung zwischen zentralen und peripheren Nebensätzen nicht relevant sind. Unter anderem können integrierte abhängige Sätze im Deutschen ins Vorfeld eines Bezugssatzes mit V2-Stellung treten, 
wie der dass-Ergänzungssatz in (21). Desintegrierte abhängige Sätze sind in dieser Position nicht zugelassen, wie der freie dass-Satz in (22) (vgl. Holler 2008: 191-207; d'Avis 2004: 147-149):

(21) Max muss sich sicher sein, dass er sich den Ferrari leisten kann.

(21a) Dass er sich den Ferrari leisten kann, muss er sich sicher sein.

(22) Max muss größenwahnsinnig sein, dass er sich jetzt den dritten Ferrari kauft.

(22a) *Dass er sich jetzt den dritten Ferrari kauft, muss er größenwahnsinnig sein.

Auch weiterführende w-Relativsätze können nicht das Vorfeld besetzen:

(23) Im Juli übernehme ich die Zahnarztpraxis, worauf ich mich sehr freue.

(23a) *Worauf ich mich sehr freue, übernehme ich im Juli die Zahnarztpraxis.

Darüber hinaus gibt es einige Arten von Adverbialsätzen, die nicht das Vorfeld ihres Bezugssatzes einnehmen können, etwa sodass- und zumal-Sätze:

(24) Ich habe meinen Schlüssel verloren, sodass ich jetzt nicht mehr hereinkomme.

(24a) *Sodass ich jetzt nicht mehr hereinkomme, habe ich meinen Schlüssel verloren.

(25) Wir sollten ihn nicht mit Fragen belästigen, zumal wir ihn kaum kennen.

(25a) *Zumal wir ihn kaum kennen, sollten wir ihn nicht mit Fragen belästigen.

Diese Beispiele legen es nahe, die Unterscheidung zwischen integriert und desintegriert, ebenso wie die zwischen zentral und peripher, auch auf Adverbialsätze anzuwenden. Das Vorfeld eines V2-Satzes gilt im Deutschen als eindeutige Integrationsposition (vgl. Frey 2011: 44). Adverbialsätze, die im Vorfeld nicht zugelassen sind, wie sodass- und zumalSätze, wären demnach desintegriert.

Manche abhängige Sätze, die im Vorfeld nicht zugelassen oder fragwürdig sind, können im Vorvorfeld stehen (vgl. d'Avis 2004). Das gilt für freie dass-Sätze wie in (26) und für weiterführende w-Relativsätze wie in (27):

(26) Dass er sich jetzt den dritten Ferrari kauft - Max muss größenwahnsinnig sein.

(27) Worauf ich mich sehr freue: Ich übernehme im Juli die Zahnarztpraxis.

Blühdorn (2012a: 204) bezeichnet die Position vor dem Vorfeld als linke externe Adjunktposition (LEA). Sodass- und zumal-Sätze sind auch von dieser Position ausgeschlossen. Pasch et al. (2003: 418-439) bezeichnen die Einleiter solcher Adverbialsätze als Postponierer:

(24b) *Sodass ich jetzt nicht mehr hereinkomme - ich habe meinen Schlüssel verloren.

(25b) *Zumal wir ihn kaum kennen - wir sollten ihn nicht mit Fragen belästigen.

Abhängige Sätze in der LEA-Position, wie in (28), beziehen typischerweise den Sprechakt, der mit dem Matrixsatz ausgeführt wird, auf den Interaktionskontext. Abhängige Sätze im Vorfeld, wie in (28a), eignen sich weit weniger für diese Funktion:

(28) Wo wir gerade davon sprechen: Es gibt ein paar neue Bilder in der Galerie.

(28a) ??Wo wir gerade davon sprechen, gibt es ein paar neue Bilder in der Galerie. 
Konopka (2006: 111) und Axel-Tober (2012: 14; vgl. auch Volodina 2011: 98-99) zeigen aber, dass illokutionsbezogene Adverbialsätze auch im Vorfeld vorkommen:

(29) Wenn Sie mich fragen: Sie sollten vorsichtig sein.

(29a) Wenn Sie mich fragen, sollten Sie vorsichtig sein.

Bei LEA-Stellung wie in (29) ist die bevorzugte Lesart illokutionsbezogen: 'mich zu fragen ist eine Bedingung, die es begünstigt, dass ich Ihnen empfehle, vorsichtig zu sein'. Bei Vorfeldstellung, wie in (29a), ist diese Lesart zwar weniger naheliegend, bleibt aber möglich. Die bevorzugte Lesart ist allerdings sachverhaltsbezogen: 'mich zu fragen ist eine Bedingung, die es nahelegt, vorsichtig zu sein (z. B. meiner Antwort zu misstrauen)'.

Es ist umstritten, wie man Beispielpaare wie (29)/(29a) hinsichtlich ihrer Syntax interpretieren soll. Eine naheliegende Sichtweise ist die, dass Adverbialsätze wie in (29) syntaktisch desintegriert, solche wie in (29a) syntaktisch integriert sind, unabhängig davon, wie sie semantisch interpretiert werden (so etwa die Darstellung bei d'Avis 2004 und Konopka 2006: 110f.). Dagegen schließt Frey (2011: 72f.) aus der Tatsache, dass im Vorfeld eines V2-Satzes auch periphere Adverbialsätze zugelassen sein können, dass das Vorfeld nur mit Einschränkung als syntaktische Integrationsposition gelten kann. Dieser Folgerung schließen wir uns nicht an. Unserer Ansicht nach muss die Schlussfolgerung lauten, dass nicht alle im Sinne Haegemans als peripher zu betrachtenden Adverbialsätze zugleich im Sinne Hollers syntaktisch desintegriert verwendet werden müssen.

\subsection{Stark integrierte, schwach integrierte und desintegrierte Adverbialsätze}

Blühdorn/Lohnstein (2012: 188-193) und Blühdorn (2012a: 202-205) unterscheiden zwischen drei Graden syntaktischer Integration bei adverbialen Nebensätzen: stark integriert (zentral), schwach integriert (peripher) und desintegriert. Adverbialsätze aller drei Integrationsgrade werden als Adjunkte gedeutet, die auf unterschiedlicher Höhe in den Strukturbaum eingefügt werden. Stark integrierte Adverbialsätze nehmen eine tiefe Adjunktposition ein. Blühdorn/Lohnstein (2012: 190f.) behandeln sie als Adjunkte zur Tempus-Phrase. Schwach integrierte Adverbialsätze nehmen eine mittelhohe Adjunktposition ein. Blühdorn/Lohnstein (2012: 190-192) behandeln sie als Adjunkte zur Modus-Phrase. Desintegrierte Adverbialsätze sind keine Konstituenten ihres Bezugssatzes (vgl. Zifonun et al. 1997: 2323-2332; Holler 2005). Blühdorn/Lohnstein (2012: 190) stellen sie als Adjunkte zur CP dar.

Die folgende Beleggruppe aus dem Deutschen zeigt vorangestellte während-Sätze als stark integrierte, schwach integrierte und desintegrierte Adverbialia:

(30) Während ich hier diese Karte schreibe, ist mein Schatz bei euch, bei seiner geliebten Familie. (stark integriert)

(31) Während ich zu Fuß gehe, fährt mein Mann immer mit dem Auto. (schwach integriert)

(32) Während ich zu Hause bleibe: Dieses Mal ist Michael weg. (desintegriert)

Die während-Sätze in (30) und (31) stehen im Vorfeld des Matrixsatzes. Sie sind dadurch als syntaktisch integriert zu erkennen. Der während-Satz in (32) ist desintegriert. Er steht in der LEA-Position. Der während-Satz in (30) kann bei gleicher deskriptiver Satzbedeutung abge- 
spalten (durch eine Spaltsatzkonstruktion vom Satzrest getrennt) werden. Er ist stark integriert:

(30a) Es ist, während ich hier diese Karte schreibe, dass mein Schatz bei euch ist, bei seiner geliebten Familie.

Der adversative während-Satz in (31) kann nicht ohne Veränderung der deskriptiven Verknüpfungsbedeutung abgespalten werden. Er ist schwach integriert:

(31a) *Es ist, während ich zu Fuß gehe, dass mein Mann immer mit dem Auto fährt.

Abspaltbarkeit eignet sich gut als Kriterium für die Unterscheidung zwischen starker und schwacher Integration.

In der folgenden Beleggruppe aus dem Italienischen dient vor allem die Interpunktion als Mittel, um die unterschiedlichen Integrationsgrade der mentre-Sätze anzuzeigen:

(33) Mentre mio figlio fa i compiti io rimango lì con lui. (stark integriert) Während mein Sohn seine Hausaufgaben macht, bleibe ich dort bei ihm.

(34) Mentre Kaoko è una ragazza normale, la sorella è così perfetta. (schwach integriert)

Während Kaoko ein normales Mädchen ist, ist ihre Schwester so perfekt.

(35) Sono sola qui davanti al computer. E mentre io sono a casa: mi chiedo dove sia lui. (desintegriert)

Ich sitze hier einsam vor dem Computer. Und während ich zu Hause bin: ich frage mich, wo ist er.

Der mentre-Satz in (35) ist durch einen Doppelpunkt von seinem Bezugssatz getrennt. Das deutet auf syntaktische Desintegration hin. Die mentre-Sätze in (33) und (34) sind Konstituenten ihrer Matrixsätze. In (34) ist der mentre-Satz durch ein Komma abgegrenzt. Das ist ein Hinweis auf schwache syntaktische Integration. In (33) steht kein Komma. Das deutet auf starke Integration hin. Der mentre-Satz in (33) ist bedeutungserhaltend abspaltbar, der in (34) nicht:

(33a) È mentre mio figlio fa i compiti che io rimango lì con lui.

Es ist, während mein Sohn seine Hausaufgaben macht, dass ich dort bei ihm bleibe.

(34a) * È mentre Kaoko è una ragazza normale che la sorella è così perfetta. Es ist, während Kaoko ein normales Mädchen ist, dass ihre Schwester so perfekt ist.

Wir werden im Folgenden (i) die Voranstellbarkeit in die Spezifizierer-Position einer C-Projektion (eine SpC-Position) und (ii) die Abspaltbarkeit als Unterscheidungskriterien für die syntaktischen Integrationsgrade von Adverbialsätzen in den Mittelpunkt stellen. Welche Positionen im Deutschen und Italienischen SpC-Positionen sind, wird in Kapitel 3 dargestellt. Stark integrierte Adverbialsätze können eine SpC-Position einnehmen und sind abspaltbar; schwach integrierte Adverbialsätze können eine SpC-Position einnehmen, sind aber nicht abspaltbar; desintegrierte Adverbialsätze können keine SpC-Position einnehmen und sind auch nicht abspaltbar (vgl. Blühdorn/Lohnstein 2012: 189). 
Darüber hinaus werden wir die These vertreten, dass zwischen den Integrationsgraden und unterschiedlich hohen Adjunktpositionen im Strukturbaum zwar prototypische Zuordnungen bestehen, wie von Blühdorn/Lohnstein (2012: 190f.) angenommen, dass diese Zuordnungen aber nicht ausnahmslos gelten. In Kapitel 4 werden wir zeigen, dass Adverbialsätze aller Integrationsgrade mehrere Positionen im Strukturbaum einnehmen können. Für jeden Integrationsgrad ist eine spezifische Kombination von Stellungsmöglichkeiten charakteristisch.

\subsection{Die semantische Hierarchie der Adverbialia nach Cinque}

Cinque (1999) hat anhand von Beobachtungen aus dem Italienischen, dem Englischen und anderen Sprachen Abfolgebeschränkungen für Adverbialia in der Linearstruktur des Satzes untersucht, insbesondere an der linken Peripherie. Nach seinen Beobachtungen lassen sich mehr als 30 semantische Klassen von Adverbialia unterscheiden, die in Bezug auf ihre Skopusbeziehungen eine Hierarchie bilden. Sprechaktbezogene, bewertende, evidenzielle und epistemische Adverbialia nehmen die oberen Ränge dieser Hierarchie ein, d. h. sie nehmen Skopus über alle rangniedrigeren Adverbialia. Temporale (Vergangenheit, Zukunft, Vorzeitigkeit) und modale (Irrealität, Notwendigkeit, Möglichkeit und andere) Adverbialia nehmen die mittleren Ränge ein. Aspektuelle Adverbialia (Durativität, Progressivität, Repetitivität und andere) finden sich auf den unteren Rängen, d.h. sie liegen im Skopus aller höherrangigen Adverbialia (vgl. detaillierter Cinque 1999: 106). Da die meisten der von Cinque aufgelisteten semantischen Adverbial-Funktionen auch in Satzform kodiert werden können, ist zu fragen, ob Cinques Vorschläge sich auch auf Adverbialsätze anwenden lassen.

Cinque postuliert Universalität für seine semantische Hierarchie. Das ist überzeugend, wenn man annimmt, dass Satzbedeutungen aus semantischen Objekten einer begrenzten Anzahl von Kategorien kompositionell erzeugt werden müssen (vgl. Blühdorn/Lohnstein 2012: 173-180). Adverbialia sind dann als Operatoren zu verstehen, die semantische Objekte mit bestimmten Eigenschaften als Operanden selegieren. Ihre Selektionsbeschränkungen erstrecken sich unter anderem darauf, welche anderen semantischen Operatoren in ihrem Skopus liegen dürfen bzw. müssen. Die gerade in diesem Bereich überwältigenden Übereinstimmungen zwischen den Sprachen legen die Vermutung nahe, dass die Kategorieninventare und Kompositionsregeln universal sind.

Cinque deutet seine Beobachtungen allerdings in erster Linie syntaktisch. Er nimmt an, dass die Abfolgebeschränkungen für Adverbialia sich daraus erklären, dass innerhalb einer in mehr als 30 Projektionen aufgespaltenen IP für jede Adverbialklasse eine eigene Position im Strukturbaum festgelegt ist. Nach seiner Vorstellung sind die Köpfe der einzelnen I-Projektionen für die Modus-, Tempus- und Aspekt-Morphologie des Verbs verantwortlich, während die Adverbialia in den Spezifizierer-Positionen Platz finden.

Mit einer solchen Annahme wäre das Abfolgeverhalten der Adverbialia möglicherweise doppelt erklärt: zum einen durch ihre hierarchische Position im Strukturbaum, zum anderen durch ihre semantische Operandenselektion. Nach unseren Beobachtungen ist die syntaktische Anordnung von Adverbialia in der Praxis aber viel weniger streng geregelt, als es Cinques syntaktische Herleitungen erwarten lassen. Für etliche seiner Abfolge-Generalisierungen geben die von uns untersuchten Daten keine konklusive Evidenz. Ein Belegpaar aus dem Italienischen: 
(36) Il duca Federico fece dotare la città da Francesco di Giorgio Martini di una potente Rocca, successivamente purtroppo demolita e di cui restano solo pochi ruderi. Herzog Federico ließ die Stadt durch Francesco di Giorgio Martini mit einer starken Burg versehen, die später leider abgerissen wurde und von der nur noch wenige Ruinen vorhanden sind.

(37) La manifestazione si svolgerà nell'Orto della Botanica inferiore nel quale vi era, sino al settecento, un edificio adibito a spezieria purtroppo successivamente demolito.

Die Veranstaltung wird im unteren Botanischen Garten stattfinden, wo es bis zum 18. Jahrhundert ein Gebäude gab, das als Gewürzlager diente und das leider später abgerissen wurde.

In (36) steht das temporale Adverb successivamente ('später') vor dem Bewertungsadverb purtroppo ('leider'). In (37) stehen die gleichen Adverbien in umgekehrter Reihenfolge. Beide Sätze sind eindeutig so zu verstehen, dass successivamente im Skopus von purtroppo liegt. Eine umgekehrte Skopusanordnung kommt nicht in Frage. In Bezug auf die syntaktische Struktur und die semantischen Relationen ist keinerlei Unterschied zwischen den Sätzen zu beobachten, d. h. die Adverbkombinationen sind gleichberechtigte Ausdrucksalternativen. Beispiele dieser Art lassen sich ohne Schwierigkeit in großer Zahl finden. (38) und (39) zeigen die deutschen Adverbien gewöhnlich (habituell) und offenbar (epistemisch) in unterschiedlichen Abfolgen. Unabhängig von der syntaktischen Anordnung nimmt offenbar auf jeden Fall Skopus über gewöhnlich:

(38) Sie ist freundlich und einfühlsam, auch wenn sie gewöhnlich offenbar eher wenige nicht allzu intensive Freundschaften pflegt.

(39) Die Kandidatin der Republikaner treibt offenbar gewöhnlich Vetternwirtschaft.

Bezieht man satzförmige Adverbialia in die Untersuchung ein, so wird das Bild noch uneinheitlicher. Adverbialsätze können zwar durchweg Positionen in der I-Domäne einnehmen, aber solche Positionen sind keineswegs immer typisch. Wenn man ihr unterschiedliches syntaktisches Verhalten im Detail untersucht (s. u. Kapitel 4), scheint es wenig plausibel, dass alle Adverbialsätze ihre Grundposition in der I-Domäne haben sollten.

Unseres Erachtens besteht für ein Modell, das das Stellungsverhalten der Adverbialia aus einer kartographischen Syntax der I-Domäne ableitet, de facto kein Bedarf. Um die für die Komposition der Satzbedeutung benötigte Skopusanordnung abzuleiten, ist die Operandenselektion gemäß der universalen semantischen Hierarchie völlig ausreichend. Es besteht eine natürliche Tendenz, in der Syntax lineare Anordnungen zu wählen, die die erwünschte semantische Skopushierarchie widerspiegeln, aber es gibt zahlreiche Faktoren (Informationsstruktur, Rhythmus, stilistische Konventionen usw.), die dazu führen können, dass andere syntaktische Linearisierungen gewählt werden. Wir folgen deshalb in der Syntax dem traditionelleren und flexibleren Adjunkt-Modell. Auf die semantische Adverbialien-Hierarchie werden wir mehrmals zurückkommen.

\section{Lineare und hierarchische Satzstruktur}

Mit Blick auf die typische Linearstruktur seiner Sätze wird das Deutsche oft als V2- bzw. Verbletzt-Sprache klassifiziert, das Italienische dagegen als SV-Sprache. Eine vergleichende Untersuchung zur Syntax von Adverbialsätzen muss ein Strukturmodell zugrundelegen, das 
diesen typologischen Unterschied überbrücken kann. Solche Modelle sind im Rahmen der generativen Grammatik entwickelt worden (für einen knappen Überblick vgl. z. B. Lohnstein 2014).

\subsection{Deutsche und italienische Sätze im Vergleich}

Deutsche Sätze werden spätestens seit Drach (1937) als Klammer- und Felderstrukturen beschrieben (vgl. Höhle 1986; Ramers 2006: 97f.): Das sogenannte Mittelfeld wird von einer linken und einer rechten Verbposition eingerahmt. Links der linken Verbposition liegt das Vorfeld, rechts der rechten Verbposition das Nachfeld. Diese Linearstruktur ist im Rahmen der generativen Syntax als Komplementiererphrase (CP) expliziert worden (vgl. Sternefeld 2008/2009: 322-325):

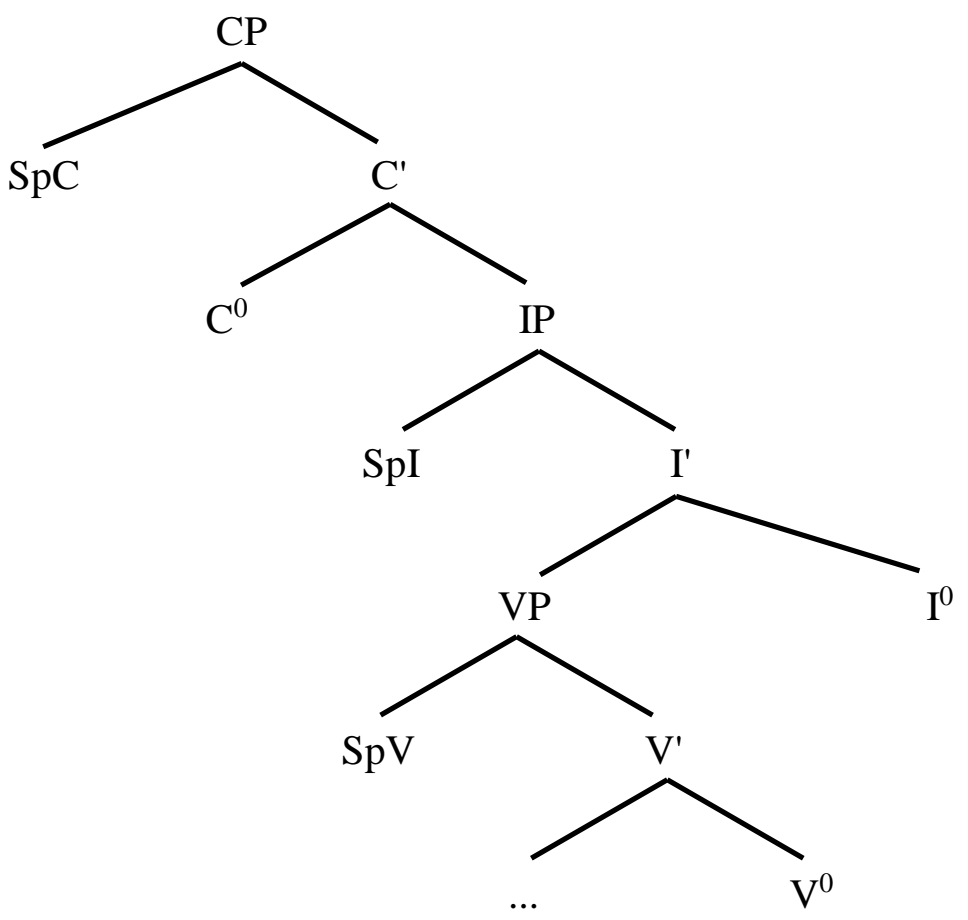

\begin{tabular}{|l|l|l|l|l|l|}
\hline Vorfeld & $\begin{array}{l}\text { linke } \\
\text { Verb- } \\
\text { pos. }\end{array}$ & Mittelfeld & \multicolumn{2}{|l|}{ rechte Verbpos. } & Nachfeld \\
\hline & dass & Hans Kartoffeln & essen & will & \\
\hline wer & will & Kartoffeln & essen & & \\
\hline wer & & Kartoffeln & & isst & \\
\hline die & & Hans & essen & will & \\
\hline Hans & hat & Kartoffeln & gegessen & & \\
\hline
\end{tabular}

Abb. 1: Strukturbaum und Linearstruktur des deutschen Satzes

Der verbale Kern des Satzes nimmt die rechte Verbposition ein. Infinite Verbteile entsprechen der $\mathrm{V}^{0}$-Position, dem Kopf der Verbalphrase. Finite Verbteile in Endstellung bilden $\mathrm{I}^{0}$, den Kopf der Flexionsphrase (IP). In Sätzen mit Endstellung des Finitums (VE) kann die linke Verbposition $\left(\mathrm{C}^{0}\right)$ mit einem Subjunktor (z. B. weil) oder einem Komplementierer (z. B. dass) besetzt sein. In Sätzen mit Erst- oder Zweitstellung des Finitums (V1 bzw. V2) nimmt dieses die linke Verbposition ein. Dann sind Subjunktoren bzw. Komplementierer ausgeschlossen. Die übrigen Satzglieder haben ihre Grundpositionen im Mittelfeld. In V2-Sätzen wird eine 
Konstituente ins Vorfeld (SpC, die Spezifizierer-Position der $\mathrm{CP}$ ) bewegt. Interrogativa in abhängigen oder unabhängigen Substitutionsfragen (w-Fragen) sowie Relativausdrücke in Relativsätzen stehen ebenfalls im Vorfeld (vgl. insgesamt Sternefeld 2008/2009: 319-434; Blühdorn/Lohnstein 2012: 180-222). Im Unterschied zum Italienischen stehen $\mathrm{V}^{0}$ und $\mathrm{I}^{0}$ im Deutschen rechts von ihren Komplementen, d. h. die Verbalphrase und die Flexionsphrase sind im Deutschen kopffinal.

Im italienischen Satz steht das Verb typischerweise rechts des Subjekts (SV). Nur in Sätzen mit sogenannter Inversion ist das Subjekt dem Verb nachgestellt. Objekte stehen immer rechts des Verbs. Adverbialia stehen typischerweise am linken oder rechten Satzrand, kommen aber auch im Satzinneren vor. Die generative Grammatik expliziert italienische Sätze als Flexionsphrasen (IP) oder Komplementiererphrasen (CP) (vgl. Müller/Riemer 1998:42-73):

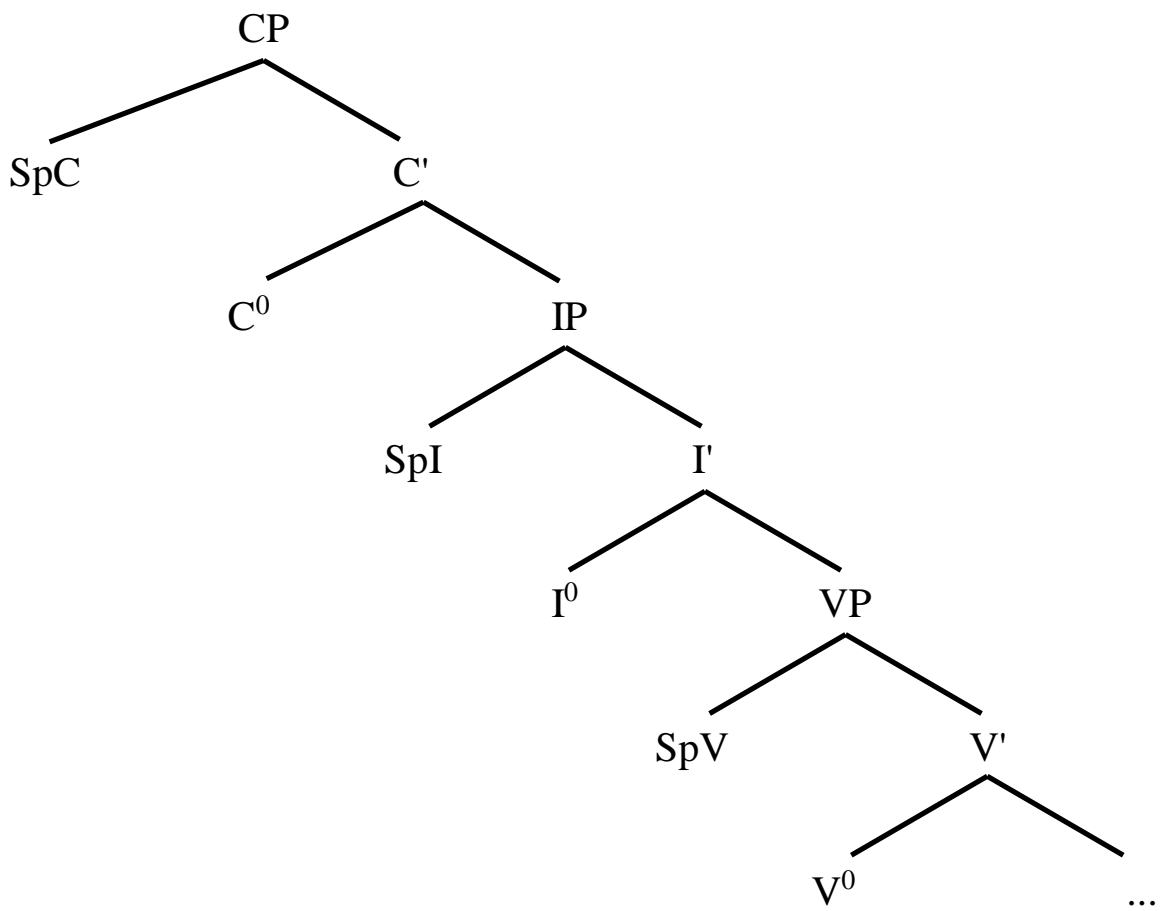

\begin{tabular}{|l|l|l|l|l|l|}
\hline $\begin{array}{l}\text { Relativa / } \\
\text { Interrogativa } \\
\text { / Topiks }\end{array}$ & $\begin{array}{l}\text { Kom- } \\
\text { plemen- } \\
\text { tierer }\end{array}$ & Subjekt & \multicolumn{4}{|l|}{ Verb } & $\begin{array}{l}\text { (Subjekt), Objekte und weitere } \\
\text { Satzglieder }\end{array}$ \\
\hline & & Greta & ha & dato & il libro a Mario \\
\hline & che & Greta & dà & & il libro a Mario \\
\hline chi & & & vuol & dare & il libro a Mario \\
\hline & che & Greta & dà & & a Mario \\
\hline a cui & & Greta & dà & & il libro \\
\hline & se & Mario & $\grave{e}$ & arrivato & \\
\hline quando & & & $\grave{e}$ & arrivato & Mario \\
\hline il libro & & Greta & lo dà & & a Mario \\
\hline
\end{tabular}

Abb. 2: Strukturbaum und Linearstruktur des italienischen Satzes

Im Italienischen steht das Verb in allen Satzarten im Zentrum des Satzes. Finite und infinite Verbformen stehen typischerweise zusammen. Nur einige Adverbien wie già oder appena können dazwischentreten. Einfache Hauptsätze, die mit dem Subjekt beginnen, werden als Flexionsphrasen (IP) analysiert. Komplementierer (z. B. che), Relativpartikeln (che) oder Subjunktoren (z. B. se) nehmen die Kopfposition $\mathrm{C}^{0}$ ein und eröffnen damit die C-Ebene (vgl. Müller/Riemer 1998: 69). Interrogativa (z. B. chi oder quando) und phrasenförmige Relativ- 
ausdrücke (z. B. a cui) nehmen auch im Italienischen eine SpC-Position ein (vgl. Renzi/Salvi/ Cardinaletti 2001, Bd. 2: 457-511; Benincà 2001: 41-50). Manche Konstituenten (Subjekt, Objekte oder Adverbialia) können zum Zwecke einer informationsstrukturellen Hervorhebung, z .B. als Topik, vorangestellt und beim Verb durch eine klitische Proform wiederaufgenommen werden (vgl. Cinque 1990: 56-97; Rizzi 1997: 285f.; Renzi/Salvi/Cardinaletti 2001, Bd. 1: 167-189; Ferrari 2012). Wir nehmen an, dass auch solche vorangestellten Konstituenten eine SpC-Position besetzen. Klitische Pronomina behandeln wir in Anlehnung an Schwarze (1995: 340f.) vereinfacht als Verbteile. $\mathrm{V}^{0}$ und $\mathrm{I}^{0}$ stehen im Italienischen links ihrer Komplemente.

Adverbialsätze können in beiden Vergleichssprachen unterschiedliche Strukturpositionen einnehmen. Wir nehmen an, dass sie Adjunkte zur VP, zur IP oder zur CP sein können. Prinzipiell können Adjunkte ihren Wirt nach rechts oder nach links erweitern. Im deutschen Satz bestehen fünf Stellungsmöglichkeiten:

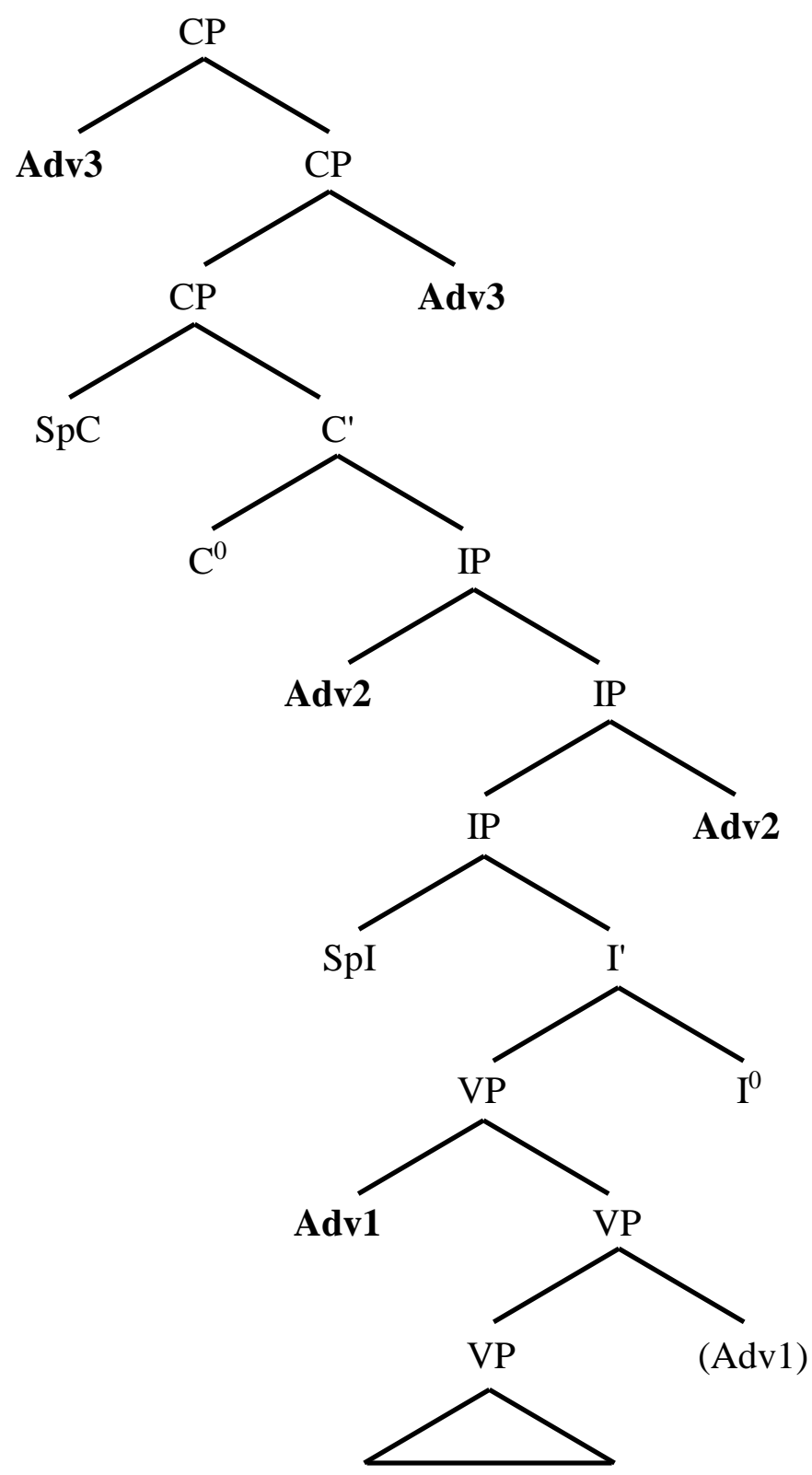

Abb. 3: Mögliche Positionen für VP-, IP- und CP-Adjunkte im deutschen Satz 
Adjunkte zur CP (Adv3) sind syntaktisch desintegriert. Sie stehen am Satzanfang als linkes externes Adjunkt (LEA) oder am Satzende als rechtes externes Adjunkt (REA). Adjunkte zur IP (Adv2) stehen am Anfang des Mittelfeldes rechts der linken Verbposition $\left(\mathrm{C}^{0}\right)$ oder am Satzende rechts der rechten Verbposition $\left(\mathrm{I}^{0}\right)$. Adjunkte zur VP (Adv1) stehen im Deutschen in der Regel links von $\mathrm{V}^{0}$ im zentralen oder rechten Mittelfeld. Rechts von $\mathrm{V}^{0}$ müssten sie im Nebensatz zwischen den infiniten und den finiten Verbteilen stehen (?weil ich ihn gesehen schon habe). Solche Ausdrücke sind fragwürdig bzw. ungrammatisch.

Im Italienischen können Adjunkte auf allen Ebenen links oder rechts ihres Wirtes stehen:

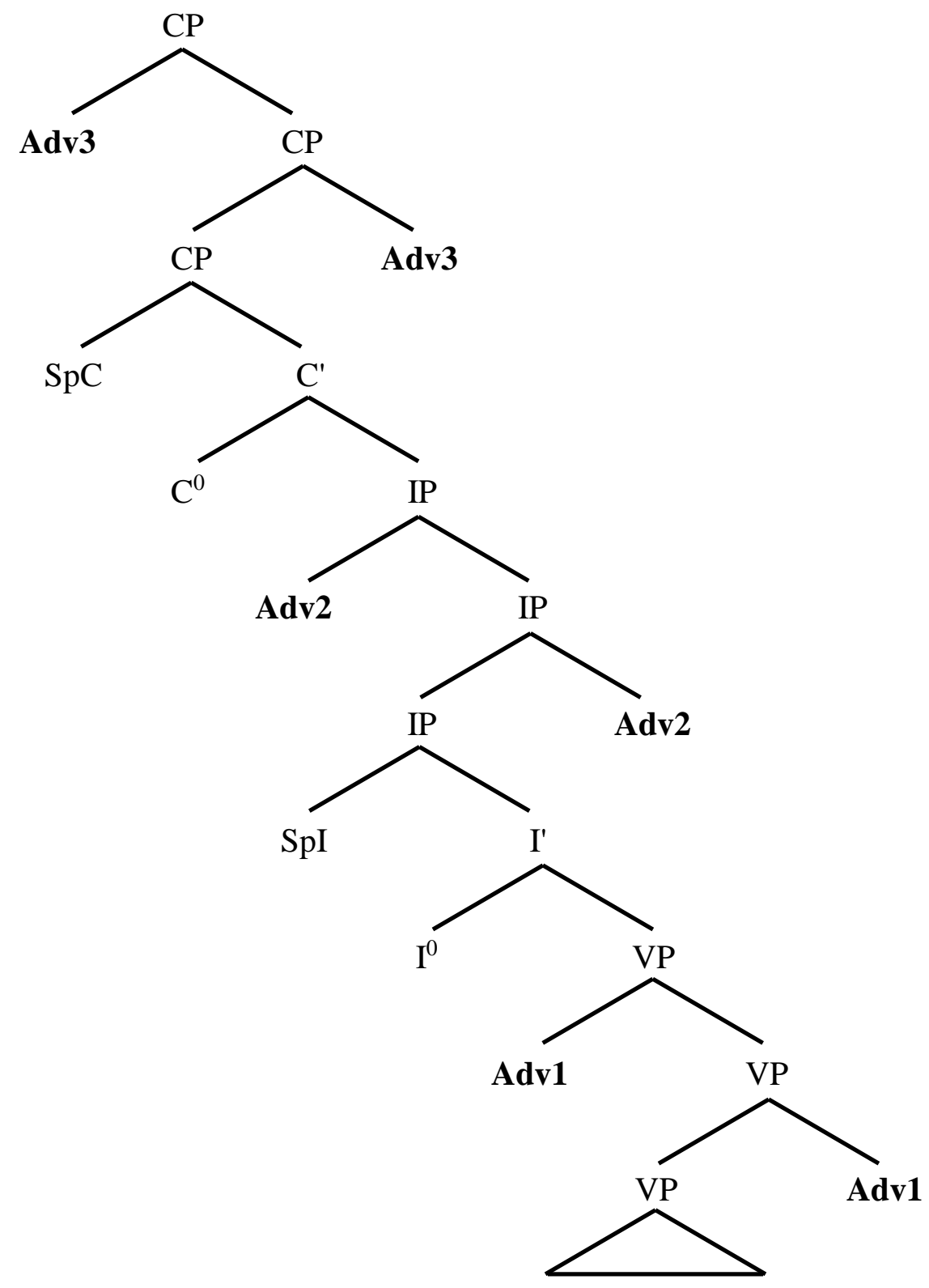

Abb. 4: Mögliche Positionen für VP-, IP- und CP-Adjunkte im italienischen Satz

Adjunkte zur CP (Adv3) stehen am linken oder rechten Satzrand. Rechte IP-Adjunkte (Adv2) stehen am Satzende. Linke IP-Adjunkte stehen am Satzanfang, wenn keine C-Ebene vorhanden ist. In Sätzen mit C-Ebene stehen sie zwischen $C^{0}$ und dem Satzrest - (il libro) che probabilmente Greta darà a Mario. Rechte VP-Adjunkte (Adv1) stehen rechts des Verbs und 
seiner Ergänzungen, also ebenfalls am Satzende, soweit keine Adjunkte in höheren Strukturpositionen folgen. Linke VP-Adjunkte stehen zwischen finitem und infinitem Verbteil.

\subsection{Adverbialia in der Linearstruktur}

Die Strukturen des deutschen und des italienischen Satzes erscheinen in dieser Darstellung als gut vergleichbar. Wir gehen nun nacheinander auf die Strukturpositionen ein, die von Adverbialia eingenommen werden können, wobei wir der Linearität des Satzes von links nach rechts folgen.

\subsubsection{Nullstelle}

An der extremen linken Satzperipherie befindet sich die sogenannte Nullstelle (vgl. Pasch et al. 2003: 70), die von koordinierenden Konjunktionen (Konjunktoren; z. B. oder, aber bzw. oppure, $m a$ ) eingenommen wird:

(40) Klar lache ich, aber Greta ist nicht der Grund dafür.

(41) Io ho traslocato, ma lei è rimasta nella nostra vecchia casa.

Ich bin umgezogen, aber sie ist in unserer alten Wohnung geblieben.

Der durch den Konjunktor eingeleitete Satz kann als dessen syntaktisches Komplement analysiert werden (vgl. Johannessen 1998: 108-113). Die Nullstelle wird gelegentlich auch von Adverbien eingenommen, die sich zu Konjunktoren weiterentwickelt haben, im Deutschen z. B. von nämlich oder also (vgl. Blühdorn 2012a: 201). Sie ist eine Kopfposition, also für Phrasen (einschließlich Sätze) nicht zugelassen. Adverbialsätze können die Nullstelle nicht einnehmen.

\subsubsection{LEA-Position}

Rechts neben der Nullstelle liegt die linke Adjunktposition zur CP (LEA-Position). Sie ist eine Position für syntaktisch desintegrierte Ausdrücke. Im Deutschen liegt sie vor dem Vorfeld (vgl. Altmann 1981: 47-50), im Italienischen vor Interrogativa und vor Konstituenten, die aus informationsstrukturellen Gründen vorangestellt sind:

(42) Es ist ökologisch unsinnig, so viel Verpackungsmaterial zu gebrauchen. Deshalb: Ich würde nie eine solche Maschine kaufen.

(43) Il Governo italiano ha dimostrato di volere accettare le regole comuni della finanza pubblica. Perciò: le basi tecniche e politiche per una iniziativa più forte a livello europeo sono già preparate.

Die italienische Regierung hat gezeigt, dass sie die gemeinsamen Regeln für die Staatsfinanzen akzeptieren will. Somit: die technischen und politischen Grundlagen für eine stärkere Initiative auf europäischer Ebene sind bereits gelegt.

Die LEA-Position kann auch von Adverbialsätzen eingenommen werden (s. u. Kapitel 4). Im Italienischen kann, wenn auf ein Adverbiale am Satzanfang wie in (43) weder eine informationsstrukturell hervorgehobene, vorangestellte Konstituente noch ein Interrogativum folgt, nicht sicher entschieden werden, ob das Adverbiale in der LEA-Position oder in der linken Adjunktposition zur IP steht. Desintegrierte Ausdrücke am Satzanfang (LEA-Ausdrücke) werden in der geschriebenen Sprache typischerweise durch Interpunktionszeichen (wie Dop- 
pelpunkte oder Gedankenstriche) vom Satzrest abgegrenzt. In der gesprochenen Sprache wird Desintegration oft durch phonetische Diskontinuitäten wie Pausen oder Tonwechsel angezeigt.

\subsubsection{SpC}

Rechts der LEA-Position liegt die SpC-Position. Sie ist eine Integrationsposition, die den Satz eröffnet, wenn die LEA-Position unbesetzt ist. Im Deutschen entspricht diese Position dem Vorfeld. In beiden Sprachen kann sie mit Interrogativa oder Relativa besetzt werden. Im Italienischen wird sie unter anderem von Konstituenten eingenommen, die aus informationsstrukturellen Gründen vorangestellt sind. Das gilt auch für vorangestellte, informationsstrukturell hervorgehobene, integrierte Adverbialia:

(44) Gestern hat er mir Rosen gebracht.

(45) Ieri mattina il pacco con dentro il tuo vestito lo ha ritirato tuo fratello. wörtl.: Gestern vormittag das Paket mit deinem Kleid drin das hat dein Bruder abgeholt.

Anders als im Deutschen können im italienischen Satz mehrere C-Projektionen übereinanderliegen, sodass mehrere SpC-Positionen zur Verfügung stehen. Dadurch können mehrere Konstituenten aus informationsstrukturellen Gründen vorangestellt werden. Rizzi (1997) unterscheidet in der C-Domäne zwischen Komplementiererphrase, Topikphrase und Fokusphrase. Diese Unterscheidung vollziehen wir hier nicht im Detail nach, unter anderem deshalb, weil im italienischen Satz auch mehrere Konstituenten als Topiks vorangestellt werden können. Die genauen syntaktischen Strukturen von Sätzen wie (45), in denen das der Fall ist, können wir hier nicht aufklären. (45) deuten wir vereinfacht so, dass die vorangestellten Konstituenten - das Zeitadverbiale ieri mattina und das Objekt il pacco con dentro il tuo vestito - die Spezifiziererpositionen übereinanderliegender C-Projektionen besetzen. Beispiel (46) enthält sogar drei übereinandergeschichtete C-Projektionen. Hier sind das temporale Adverbiale lo scorso anno und das Subjekt Sabrina als Topiks vorangestellt. Beide stehen vor dem Interrogativum perché, das seinerseits ebenfalls eine SpC-Position innehat:

(46) Lo scorso anno Sabrina perché lavorava ancora lì, se non le piaceva quello che faceva?

wörtl.: Letztes Jahr Sabrina warum arbeitete sie noch da, wenn ihr nicht gefiel, was sie machte?

In Sätzen wie (45) und (46) könnte man alternativ auch annehmen, dass die vorangestellten Adverbialia die LEA-Position einnehmen. Eine solche Annahme scheint uns für Zeitadverbialia, die nicht den Sprechakt, sondern den beschriebenen Sachverhalt situieren, aber nicht plausibel. Ihre Voranstellung erfolgt ebenso wie die von Argumenten aus informationsstrukturellen Gründen und sollte deshalb eine SpC-Position zum Ziel haben.

Im Unterschied zur LEA-Position sind SpC-Positionen nur für syntaktisch integrierte Konstituenten zugänglich. Die folgenden Beispiele zeigen vorangestellte Adverbialsätze in SpCPositionen - stark integriert (abspaltbar) in (47) und (49), schwach integriert (nicht-abspaltbar) in (48) und (50): 
(47) Als die WM im Ringen stattfand, war es von vornherein klar, dass der Endkampf zwischen einem Amerikaner und einem Russen ausgetragen würde.

(47a) Es war, als die WM im Ringen stattfand, dass es von vornherein klar war, dass der Endkampf zwischen einem Amerikaner und einem Russen ausgetragen würde.

(48) Da das Headset sich nicht zusammenklappen lässt, braucht es schon ein bisschen Stauraum.

(48a) *Es ist, da das Headset sich nicht zusammenklappen lässt, dass es schon ein bisschen Stauraum braucht.

(49) Quando Magda era a casa il bucato lo faceva sempre lei. wörtl.: Als Magda zu Hause war, die Wäsche die wusch immer sie.

(49a) È quando Magda era a casa che il bucato lo faceva sempre lei.

(50) Mentre i figli di Sara brontolano spesso, i capricci i miei figli non li fanno quasi mai.

wörtl.: Während Saras Kinder häufig brummen, Zicken meine Kinder haben sie fast nie.

(50a) *È mentre i figli di Sara brontolano spesso che i capricci i miei figli non li fanno quasi mai.

SpC-Positionen können nicht von desintegrierten Ausdrücken, z. B. satzbezogenen weiterführenden Relativsätzen wie in (51) oder finalen dass- bzw. che-Sätzen wie in (52) eingenommen werden:

(51) Ich bin kein Jurist, weshalb meine Ratschläge mit Vorsicht zu genießen sind.

(51a) *Weshalb meine Ratschläge mit Vorsicht zu genießen sind, bin ich kein Jurist.

(52) Mia figlia la aiuto sempre io, che faccia bene i compiti.

wörtl.: Meiner Tochter der helfe immer ich, dass sie ihre Hausaufgaben richtig macht.

(52a) * Che faccia bene i compiti mia figlia la aiuto sempre io.

Gleiches gilt für desintegrierte Adverbialsätze, etwa für zumal- bzw. tanto più che-Sätze (s. o. Kapitel 2):

(53) Questo punto lo possiamo discutere con calma, tanto più che siamo sostanzialmente d'accordo.

wörtl.: Diesen Punkt den können wir in Ruhe besprechen, zumal wir uns im Prinzip darüber einig sind.

(53a) *Tanto più che siamo sostanzialmente d'accordo questo punto lo possiamo discutere con calma.

\subsubsection{Nacherstposition}

Rechts der SpC-Position (zwischen SpC und $C^{\prime}$ ) liegt die sogenannte Nacherstposition (vgl. Pasch et al. 2003: 71f.). Sie ist eine Parenthese- bzw. Desintegrationsposition, die mit einem Adverbiale besetzt werden kann, wenn die SpC-Position von einer informationsstrukturell 
hervorgehobenen Konstituente eingenommen wird (vgl. Blühdorn 2011: 280f.; Blühdorn 2012a: 202). Somit liegt die Nacherstposition niemals am Satzanfang:

(54) \{Das Buch hat er auf Deutsch gelesen. $\}$ den /FILM aber hat er auf ENGVlisch gesehen ${ }^{4}$

Im Italienischen ist ein Adverbiale als Nacherst-Parenthese zu deuten, wenn links von ihm ein informationsstrukturell hervorgehobenes Argument, insbesondere ein Objekt, steht:

(55) il /LIbro probabilmente riesco a restitu/IRtelo solo doMA Ini wörtl.: das /BUCH wahrscheinlich ich kann es dir zu/RÜCKgeben erst MOR\gen

Hierarchiehohe Adverbialia (Sprechakt- oder Bewertungsadverbialia, evidenzielle oder epistemische Adverbialia) sind besonders typisch für die Nacherstposition, aber hierarchieniedere, vor allem satzförmige Adverbialia, sind in dieser Position nicht ausgeschlossen, d. h. auch solche Adverbialia können syntaktisch desintegriert verwendet werden:

(56) Der Hund, als er das sah, sprang auf die Katze zu und wollte ihr den Kuchen entreißen.

(57) Al mio capo, quando facevo pratica, piaceva molto Mina. In macchina la mattina quando si andava in Tribunale, mentre cercavo di dirgli quello che c'era da fare, lui ascoltava Mina.

wörtl.: Meinem Chef, als ich Praktikum machte, gefiel besonders Mina. Im Auto morgens, wenn wir zum Gericht fuhren, während ich versuchte, ihm zu sagen, was erledigt werden musste, hörte er Mina.

\subsubsection{Linke Adjunktposition zur IP}

Rechts der Nacherstposition liegt die linke Adjunktposition zur IP. Sie ist eine Standardposition für schwach integrierte Adverbialia. Im Deutschen liegt sie im linken Mittelfeld, im Italienischen vor dem Subjekt, wenn dieses nicht als Topik vorangestellt ist, also häufig am Satzanfang:

(58) Ich verwende, da ich es mir leisten kann, meine freie Zeit, um Artikel zu schreiben.

(59) Mentre io sono piccola e debole, le altre sono grandi e potenti.

Während ich klein und schwach bin, sind die anderen groß und kräftig.

Auch stark integrierte (abspaltbare) Adverbialia können in der linken Adjunktposition zur IP stehen:

(60) Bei mir werden, nachdem ich das Template geändert habe, keine E-Mails mehr abgerufen.

(61) Prima che partisse per l'Africa, Luca l'aveva portata con sé a Torino.

Bevor sie nach Afrika aufbrach, hatte Luca sie mit nach Turin genommen.

\footnotetext{
${ }^{4}$ Zum besseren Verständnis haben wir in (54) und (55) die Intonation eingetragen, mit der sie auszusprechen wären. Konventionen für die Wiedergabe der prosodischen Gestaltung: akzentuierte Silben - Großbuchstaben; unakzentuierte Silben - Kleinbuchstaben; steigender Schrägstrich vor der Akzentsilbe - steigende Tonbewegung; fallender Schrägstrich nach der Akzentsilbe - fallende Tonbewegung. Für das Italienische verwenden wir einfachheitshalber die gleiche Notation. Für unsere Zwecke ist das schadlos möglich, obgleich die italienische Intonation sich in vieler Hinsicht von der deutschen unterscheidet.
} 
Im Italienischen ist es nicht immer möglich, eindeutig zwischen desintegrierten Adverbialsätzen in der LEA-Position, integrierten Adverbialsätzen in der SpC-Position und integrierten Adverbialsätzen in der linken Adjunktposition zur IP zu unterscheiden. Sie alle können am Satzanfang stehen. Desintegrierte Ausdrücke werden in der geschriebenen Sprache typischerweise durch Interpunktionszeichen (Doppelpunkte oder Gedankenstriche) vom Satzrest abgetrennt. Schwach integrierte Ausdrücke werden häufig durch ein Komma abgegrenzt. In beiden Fällen sind die Interpunktionszeichen aber nicht obligatorisch bzw. in Belegen de facto nicht immer vorhanden. In der gesprochenen Sprache werden oft phonetische Diskontinuitäten wie Pausen oder Tonwechsel zur Anzeige syntaktischer Desintegration verwendet. Insgesamt sind solche Hinweise wichtig, aber nicht hinreichend als Kriterien für die syntaktische Analyse.

\subsubsection{Linke Adjunktposition zur VP}

Rechts der linken Adjunktposition zur IP liegt die linke Adjunktposition zur VP. Sie ist eine Standardposition für stark integrierte Adverbialia. Im Deutschen liegt sie im mittleren Mittelfeld, im Italienischen zwischen Subjekt und Verb: ${ }^{5}$

(62) Übrigens habe ich, als ich meine Ausbildung zum Rettungssanitäter abgeschlossen hatte, eine Sprachtherapie begonnen.

(63) Io quando loro si ammalano non dormo. wörtl.: Ich wenn sie krank werden schlafe nicht.

Wenn in dieser Position nicht-abspaltbare Adverbialia auftreten, sind sie Parenthesen:

(64) In diesem Fall habe ich - zumal ich unter Zeitdruck stand - alles genommen, was mir auf die Schnelle einfiel.

(65) Io, tanto più che quel libro costa molto meno, concordo con quello che hai scritto: compro quello!

Ich, zumal dieses Buch viel weniger kostet, stimme mit dem überein, was $d u$ geschrieben hast: Ich kaufe dieses!

\subsubsection{Rechte Adjunktposition zur VP}

Rechts der Objekte folgt im italienischen Satz die rechte Adjunktposition zur VP. Sie ist ebenfalls eine Standardposition für stark integrierte Adverbialia:

(66) Miei conoscenti hanno comprato già da qualche anno una casa nella zona dall'altra parte del Tevere perché lì gli alloggi costano molto meno.

Meine Bekannten haben schon vor einigen Jahren ein Haus im Stadtteil auf der anderen Seite des Tibers gekauft, weil die Wohnungen da viel weniger kosten.

Im Deutschen liegt die rechte Adjunktposition zur VP zwischen dem infiniten und dem finiten Verbteil im Nebensatz und ist nur marginal besetzbar (s.o. Abschnitt 3.1).

\footnotetext{
5 Auch zwischen mehreren Objekten sowie zwischen Objekt und Verb können stark integrierte Adverbialia auftreten. Diese Möglichkeiten blenden wir hier einfachheitshalber aus.
} 


\subsubsection{Rechte Adjunktposition zur IP}

Wenn am Satzende nicht-abspaltbare Adverbialia stehen, betrachten wir sie nicht als Adjunkte zur VP, sondern als rechte Adjunkte zur IP oder zur CP. Die rechte Adjunktposition zur IP ist eine Standardposition für schwach integrierte Adverbialia. Im Deutschen wie im Italienischen liegt sie am Satzende, im Deutschen rechts der rechten Verbposition. Sie wird üblicherweise als Nachfeld bezeichnet:

(67) Für mich sind die Segeltouren auf der Ostsee zu Ende, während meine Frau noch mit Begeisterung am Steuer stehen kann.

(68) Milo adora le scimmie nello zoo, mentre io sono sempre affascinata dall'eleganza delle giraffe.

Milo liebt die Affen im Zoo, während ich immer von der Eleganz der Giraffen fasziniert bin.

Im Deutschen können auch stark integrierte (abspaltbare) Adverbialia, insbesondere wenn sie satzförmig sind, im Nachfeld stehen (Ausklammerung):

(69) Der Bankräuber flüchtete, weil die Angestellte sich nicht regte.

(70) Es war, weil die Angestellte sich nicht regte, dass der Bankräuber flüchtete.

\subsubsection{REA-Position}

Ganz rechts außen liegt die rechte Adjunktposition zur CP (REA-Position). Sie ist eine Desintegrationsposition. In der germanistischen Literatur wird sie auch als Nachnachfeld bzw. als rechtes Außenfeld bezeichnet (vgl. Zifonun et al. 1997: 1644-1675). In dieser Position stehen Adverbialsätze, die nicht voranstellbar sind, wie in (71), und solche, die bei Voranstellung die LEA-Position einnehmen müssten, wie in (72):

(71) Die Nierenwerte waren im nicht mehr messbaren Bereich, sodass uns keine Wahl mehr blieb.

(72) Ieri sono andata finalmente a trovare Claudia nella sua nuova casa, nel tardo pomeriggio, se posso dirvelo.

Gestern habe ich Claudia endlich in ihrer neuen Wohnung besucht, am späten Nachmittag, wenn ich es euch sagen darf.

(72a) Se posso dirvelo: Ieri sono andata finalmente a trovare Claudia nella sua nuova casa, nel tardo pomeriggio.

In (72) folgt der desintegrierte Konditionalsatz (rechtes Adjunkt zur CP) auf ein Zeitadverbiale: nel tardo pomeriggio (rechtes Adjunkt zur VP, da abspaltbar). Auch Adverbialsätze, die gewöhnlich integriert auftreten, können desintegriert in der REA-Position stehen - als Nachträge im Sinne von Altmann (1981: 70-72). Hinweise auf beabsichtigte Desintegration werden in der geschriebenen Sprache durch die Interpunktion, in der gesprochenen durch die Prosodie gegeben (vgl. d'Avis 2004: 149):

(73) Ein Grieche wurde jetzt von einem Athener Gericht zu einer Haftstrafe von drei Monaten verurteilt - weil er zwei gefälschte CDs kaufte. 
(74) Troverete riposo per le vostre anime. Poiché il mio giogo è soave e il mio peso è leggero.

Ihr werdet Ruhe für eure Seelen finden. Weil mein Joch süß ist und mein Gewicht leicht.

\section{$4 \quad$ Ausgewählte Subjunktorsätze im Deutschen und Italienischen}

Wir untersuchen nun das genaue Stellungsverhalten von zehn Gruppen von Adverbialsätzen: als-, während-, wenn-, obwohl- und weil-Sätzen im Deutschen sowie quando-, mentre-, se-, sebbene- und perché-Sätzen im Italienischen. Unterschiedliche semantische Lesarten dieser Sätze berücksichtigen wir, soweit sie mit Unterschieden im syntaktischen Verhalten einhergehen. Aus Platzgründen führen wir die Anwendung der in Kapitel 2 und 3 vorgestellten Testverfahren teilweise nur kursorisch vor.

\subsection{Als- und quando-Sätze}

Als und quando leiten temporale Adverbialsätze ein (zu als vgl. Blühdorn 2003). Innerhalb dieser Sätze stehen sie wie alle Subjunktoren in der $\mathrm{C}^{0}$-Position. ${ }^{6} \mathrm{Als}$-Sätze können das Vorfeld ihres Matrixsatzes einnehmen wie in (75); quando-Sätze können als Topiks ihrem Matrixsatz vorangestellt werden wie in (76):

(75) Als ich diesen Satz las, fiel mir spontan Mielkes Rede vor der Volkskammer ein.

(76) Quando Giorgio aveva più o meno la tua età, il letto se lo faceva già da solo! wörtl.: Als Giorgio ungefähr in deinem Alter war, sein Bett das machte er schon allein!

In beiden Fällen besetzen die Adverbialsätze SpC-Positionen des Matrixsatzes. Im Italienischen verwenden wir für diese Position Belege mit Voranstellung des Adverbialsatzes vor ein seinerseits vorangestelltes Objekt, um Verwechslungen mit der linken Adjunktposition zur IP zu vermeiden (s.o. Kapitel 3).

Als- und quando-Sätze wie in (75) und (76) können abgespalten werden, sind also stark integriert:

(75a) Es war, als ich diesen Satz las, dass mir spontan Mielkes Rede vor der Volkskammer einfiel.

(76a) Era quando Giorgio aveva più o meno la tua età, che il letto se lo faceva già da solo!

Sie können sämtliche Integrationspositionen im Matrixsatz einnehmen, d.h. unter anderem die linken und rechten Adjunktpositionen zur VP und zur IP. Im Deutschen ist die rechte Adjunktposition zur VP nicht für Adverbialsätze zugelassen. Für das Italienische nehmen wir an, dass stark integrierte Adverbialia bei Nachstellung VP-Adjunkte sind. Zuerst drei deutsche dann drei italienische Belege:

(77) Ich war, als Sie kamen, im Begriff, das Café zu verlassen. (linkes IP-Adjunkt)

\footnotetext{
6 Beide kommen daneben als Relativausdrücke, quando auch als Interrogativum vor (vgl. Blühdorn/Lohnstein 2012: 204; Renzi/Salvi/Cardinaletti 2001, Bd. 1: 504f.). In diesen Funktionen stehen sie in SpC.
} 
(78) Andrea hat dem Chef, als er gestern kam, die erfreuliche Nachricht überbracht. (linkes VP-Adjunkt)

(79) Wir haben Bärlauch mitgenommen, als wir nach Frankreich zogen. (rechtes IPAdjunkt)

(80) Quando avevo più o meno 23 anni, io ero veramente un tipo! (linkes IP-Adjunkt) Als ich ungefähr 23 Jahre alt war, war ich echt ein Typ!

(81) Il mio gatto, quando c'è molto stress a casa, è nervoso. (linkes VP-Adjunkt) wörtl.: Meine Katze, wenn es gibt viel Stress zu Hause, ist unruhig.

(82) Non facevano parte di un esercito regolare quando sono stati catturati. (rechtes VP-Adjunkt)

Sie gehörten keiner regulären Armee an, als sie gefangen genommen wurden.

Als- und quando-Sätze können auch desintegriert in der REA-Position stehen:

(83) Sie waren in der Mitte der Höhle angekommen, als völlig unerwartet eine riesige Nebelwolke sie umschloss.

(84) Stavo scendendo in cantina, quando improvvisamente sentii un rumore. Ich ging gerade in den Keller hinunter, als ich plötzlich ein Geräusch hörte.

Zifonun et al. (1997: 2325f.) bezeichnen als-Sätze wie in (83) als weiterführend. Semantisch besteht ihre Besonderheit darin, dass sie nicht wie gewöhnliche Temporalsätze einen zeitlichen Kontext für den im Matrixsatz beschriebenen Sachverhalt angeben, sondern einen zeitlich nachfolgenden Sachverhalt beschreiben, für den gerade umgekehrt der Matrixsatz den Kontext liefert. Die Beschreibung lässt sich auch auf den quando-Satz in (84) anwenden. Stellt man solche als- bzw. quando-Sätze voran in eine Integrationsposition, so kehrt sich die Kontextualisierungsrelation um:

(83a) Als völlig unerwartet eine riesige Nebelwolke sie umschloss, waren sie in der Mitte der Höhle angekommen. (als-Satz im Vorfeld)

(84a) Quando improvvisamente sentii un rumore, stavo scendendo in cantina. ${ }^{7}$ (quandoSatz in der linken IP-Adjunkt-Position)

Desintegrierte als- und quando-Sätze kommen in informeller (vor allem wohl in mündlicher) Rede auch in ihrer gewöhnlichen temporal-kontextualisierenden Lesart vor, entweder vorangestellt (LEA-Position) wie in (85) oder als Nachtrag (REA-Position) wie in (86):

(85) Als ich das sah: ein großer Stein fiel mir vom Herzen.

(86) Per il nostro pianeta sarà purtroppo troppo troppo tardi. Quando l'uomo capirà finalmente di non poter vivere senza la natura.

Für unseren Planeten wird es leider viel viel zu spät sein. Wenn der Mensch endlich versteht, dass er ohne die Natur nicht leben kann.

\footnotetext{
${ }^{7}$ Für Leser, die an der Grammatikalität dieses Satzes zweifeln, hier ein geeigneter Kontext: Zeuge: ... e poi improvvisamente sentii un rumore. ('... und dann hörte ich plötzlich ein Geräusch.') - Polizist: Che cosa faceva nel momento in cui ha sentito improvvisamente un rumore? ('Was machten Sie da gerade, als Sie plötzlich ein Geräusch hörten?') - Zeuge: Quando improvvisamente sentii un rumore, stavo scendendo in cantina. ('Als ich plötzlich ein Geräusch hörte, ging ich gerade in den Keller hinunter.')
} 
Desintegrierte Verwendungen als Nacherst-Parenthesen sind bei als- und quando-Sätzen ebenfalls möglich, in schriftlichen Daten aber selten. ${ }^{8}$ Im Italienischen verwenden wir für die Nacherstposition Belege, in denen der Adverbialsatz auf ein vorangestelltes Objekt folgt, um Verwechslungen mit linken VP-Adjunkten zu vermeiden (s.o. Abschnitte 3.2.4 und 3.2.6):

(87) Das Haus, als wir ankamen, war total schmutzig!

(88) A mio figlio, quando aveva dodici anni, avevo comprato la classica "Fattoria degli animali".

Meinem Sohn, als er zwölf Jahre alt war, hatte ich die berühmte "Farm der Tiere" gekauft.

\subsection{Während- und mentre-Sätze}

Während und mentre (vgl. Blühdorn/Ravetto 2012) leiten temporale oder adversative Adverbialsätze ein (zu während vgl. Clément 1998; Lohnstein 2004: 154-158; Blühdorn 2008a: 236-239; zu mentre Bianco 2008). Beide können SpC-Positionen, also im Deutschen das Vorfeld, im Italienischen die Position eines vorangestellten Topiks, einnehmen:

(89) Während die Welt über den Zaun debattiert, begräbt Israel seine Toten. (temporal und/oder adversativ lesbar)

(90) Während ich in vielerlei Hinsicht absolut sachlich und rational sein kann, bin ich eine zarte Feder bei Tieren und gebe es auch gerne zu. (nur adversativ lesbar)

(91) Mentre sono in giro, la mia casella di posta la può aprire anche il mio compagno. (nur temporal)

wörtl.: Während ich unterwegs bin, meinen Posteingang den kann auch mein Freund überprüfen.

(92) Mentre io faccio le pulizie ogni sera, l'immondizia lui la porta solo qualche volta giù. (nur adversativ)

wörtl.: Während ich jeden Abend sauber mache, den Müll den bringt er nur manchmal runter.

Temporale während- und mentre-Sätze können abgespalten werden, sind also stark integriert (vgl. Clément 1998: 55; Blühdorn / Ravetto 2012: 54-55):

(89a) Es ist, während die Welt über den Zaun debattiert, dass Israel seine Toten begräbt. (nur temporal lesbar)

(91a) È mentre io sono in giro, che la mia casella di posta la può aprire anche il mio compagno.

Adversative während- und mentre-Sätze können nicht abgespalten werden. Sie sind schwach integriert (vgl. ebd.):

\footnotetext{
8 Unsere Belegsammlungen sind zu klein, um präzise Angaben zur Vorkommenshäufigkeit der einzelnen Stellungsvarianten zu ermöglichen. Wir haben aber nach allen beschriebenen Stellungen von Adverbialsätzen mit jeder der zehn Konjunktionen gezielt im Internet gesucht und haben dabei teilweise recht deutlich erkennen können, welche Stellungen häufig und welche selten vorkommen. Diesbezügliche (informelle) Beobachtungen nehmen wir, soweit es sinnvoll erscheint, in unsere Darstellung auf.
} 
(90a) *Es ist, während ich in vielerlei Hinsicht absolut sachlich und rational sein kann, dass ich eine zarte Feder bei Tieren bin.

$(92 a) * \grave{E}$ mentre io faccio le pulizie ogni sera che l'immondizia lui la porta solo qualche volta giù.

Während- und mentre-Sätze können alle integrierten Adjunktpositionen einnehmen:

(93) Er hat, während ich am Gepäckband auf seinen Koffer warte, nur Augen für sie. (linkes IP-Adjunkt; temporal und/oder adversativ lesbar)

(94) Ich habe ihn, während sie in Bodenwerder weinte, andauernd lächeln sehen. (linkes VP-Adjunkt; temporal und/oder adversativ)

(95) Die Türkei kann nicht einem europäischen Staat mit Krieg drohen, während wir darüber reden, sie in die EU zu lassen. (rechtes IP-Adjunkt; temporal und/oder adversativ)

(96) Mentre lui cammina, io corro come una pazza. (linkes IP-Adjunkt; temporal und/oder adversativ)

Während er geht, renne ich wie eine Verrückte.

(97) I miei fratelli, mentre io dovevo starmene in camera, potevano giocare all'aperto. (linkes VP-Adjunkt; temporal und/oder adversativ)

Meine Geschwister, während ich in meinem Zimmer bleiben musste, durften im Freien spielen.

(98) Un'auto si incendia mentre transita sulla A5. (rechtes VP-Adjunkt; nur temporal) Ein Auto gerät in Brand, während es auf der A5 fährt.

(99) In passato molti suonatori reggevano il barbat tenendo le gambe incrociate, mentre ora preferiscono adottare la posizione tipica dei chitarristi classici. (rechtes IPAdjunkt; nur adversativ)

In der Vergangenheit hielten viele Spieler den Barbat mit gekreuzten Beinen, während sie jetzt lieber die typische Haltung von klassischen Gitarristen einnehmen.

Nachgestellte temporale mentre-Sätze analysieren wir, da sie stark integriert sind, als VPAdjunkte, nachgestellte adversative mentre-Sätze, da sie schwach integriert sind, als IPAdjunkte. In den linken Adjunktpositionen zur VP kommen adversative während- und mentre-Sätze nur selten vor. In dieser Position bekommen sie Parenthese-Charakter, so z. B. in (94) und (97) bei adversativer Lesart. Während-Sätze sind als linke IP-Adjunkte wie in (93) ebenfalls selten, haben in dieser Position nach unserem Eindruck aber keinen parenthetischen Charakter. Mentre-Sätze kommen als linke IP-Adjunkte wie in (96) recht häufig vor. Der mentre-Satz in (96) könnte eventuell alternativ als Spezifizierer einer C-Projektion gedeutet werden, aber nur wenn er prosodisch und damit informationsstrukturell hervorgehoben ist.

Desintegrierte Stellungen von während- und mentre-Sätzen sind in schriftlichen Daten selten. LEA-Stellung ist nur in informeller Rede zu erwarten, bei adversativer Lesart eher als bei temporaler. Ein Beispiel aus dem Italienischen ist (100). Ähnliche Belege lassen sich auch für während finden: 
(100) Mentre noi cerchiamo di costruire il mondo che vogliamo verso uno sviluppo sostenibile: dall'altra parte della Terra gli sprechi continuano!

Während wir versuchen, die Welt aufzubauen, die wir uns wünschen, in Richtung einer nachhaltigen Entwicklung: Auf der anderen Seite der Erde geht die Verschwendung weiter!

Am Satzende werden adversative während- und mentre-Sätze gelegentlich durch einen Gedankenstrich oder Punkt abgegrenzt, was als Hinweis auf REA-Stellung gewertet werden kann. Ein Beispiel aus dem Deutschen ist (101). Werden solche während- und mentre-Sätze temporal gelesen, so fungieren sie als Nachtrag:

(101) Der neue Wahnsinn in Washington: Amerikanische Bürger müssen jetzt ihre Waffen abgeben - während die Regierung Massen von Sturmgewehren und Milliarden Schuss Munition hortet.

Während-Sätze als Nacherst-Parenthesen sind sowohl in temporaler als auch in adversativer Lesart marginal. Mentre-Sätze kommen als Nacherst-Parenthesen vor:

(102) L'aria condizionata, mentre sono tutti in vacanza, non l'accendono mai nei nostri uffici.

Die Klimaanlage, während alle in Urlaub sind, schalten sie in unseren Büros niemals an.

\subsection{Wenn- und se-Sätze}

Wenn und se leiten konditionale Adverbialsätze ein. Diese können Bedingungen angeben, unter denen der im Matrixsatz beschriebene Sachverhalt faktisch wird (Faktizitätsbedingungen), Bedingungen, unter denen die mit dem Matrixsatz gemachte Aussage wahr ist (epistemische Bedingungen/Wahrheitsbedingungen), oder Bedingungen, die den mit dem Matrixsatz ausgeführten Sprechakt kontextualisieren (illokutionäre Bedingungen; vgl. Sweetser 1990: 113-121; Zifonun et al. 1997: 2242, 2326-2332; Günthner 1999b; Haegeman 2003; d'Avis 2004: 150f.; Haegeman 2006; Blühdorn 2008a; Reis/Wöllstein 2010: 124). Diese Verwendungsweisen lassen sich unschwer in Cinques semantische Hierarchie der Adverbialia (s.o. Abschnitt 2.4) einordnen. Konditionalsätze mit Sachverhaltsbezug gehören zum mittleren, epistemische und sprechaktbezogene zum oberen Hierarchiebereich. Wenn- und se-Sätze aller drei Verknüpfungsebenen können SpC-Positionen einnehmen. Drei Beispiele aus dem Deutschen:

(103) Wenn ich um mich schlage, werde ich von meinen Eltern geliebt. (Faktizitätsbedingung)

(104) Wenn ich es richtig verstanden habe, wurde Kindergeld zurückgefordert. (epistemische Bedingung)

(105) Wenn ich es so sagen darf, hat Rapid vor diesem Spiel die Hosen voll gehabt. (illokutionäre Bedingung)

Für sprechaktverknüpfende Konditionalsätze ist Vorfeldstellung wie in (105) aber eher untypisch (vgl. Konopka 2006: 111; Volodina 2011: 98f;; Axel-Tober 2012: 14). Typischer ist desintegrierte Stellung in der LEA-Position (s.o. Abschnitt 2.2; vgl. d'Avis 2004: 150): 
(105a) Wenn ich es so sagen darf: Rapid hat vor diesem Spiel die Hosen voll gehabt.

Drei Beispiele aus dem Italienischen mit se-Sätzen in SpC-Position:

(106) Se vinco la partita, la cena te la pago io. (Faktizitätsbedingung) wörtl.: Wenn ich das Spiel gewinne, dein Abendessen das bezahle dir ich.

(107) Se ho capito bene, il computer non lo tocchi da anni. (epistemische Bedingung) wörtl.: Wenn ich es richtig verstanden habe, den Computer den rührst du nicht an seit Jahren.

(108) Okay, se insisti, tutta questa storia l'ho saputa ieri da tua madre. (illokutionäre Bedingung)

wörtl.: Okay, wenn du darauf bestehst, diese ganze Geschichte die habe ich erfahren gestern von deiner Mutter.

Konditionalsätze mit illokutionärer Lesart in SpC-Position wie in (108) sind im Italienischen nicht untypisch, da der Unterschied zwischen LEA- und SpC-Position an der Satzoberfläche kaum zu erkennen ist. Würde man in (108) anstelle des Kommas einen Doppelpunkt setzen, so könnte man die Interpunktion als Hinweis auf beabsichtigte Desintegration (LEA-Stellung) deuten (vgl. Volodina 2011: 205).

Sachverhaltsbezogene Konditionalsätze sind stark integriert; sie können abgespalten werden wie in (103a) und (106a). Wahrheits- und sprechaktbezogene Konditionalsätze sind schwach integriert oder desintegriert; sie erlauben keine Abspaltung. Die Konditionalsätze in $(104 a) /(105 b)$ und (107a)/(108a) könnten nur für Faktizitätsbedingungen stehen, was zu unplausiblen oder sinnlosen Interpretationen führen würde: ${ }^{9}$

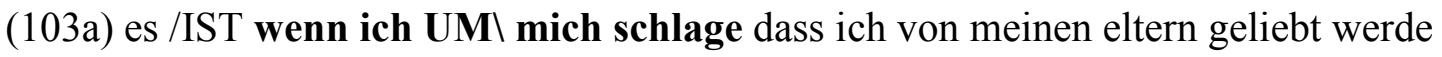

(106a) è se /VINco la parTIIta che la cena te la pago io

(104a) ?es /IST wenn ich es richtig verSTANlden habe dass kindergeld zurückgefordert wurde

(105b) ?es /IST wenn ich es so SAlgen darf dass rapid vor dem spiel die hosen voll hatte

(107a) ?è se ho capito /BE $/ n e$ che il computer non lo tocchi da anni

(108a) ?è se in/SI/sti che tutta questa storia l'ho saputa ieri da tua madre

Sachverhaltsbezogene Konditionalsätze kommen in allen Integrationspositionen vor (vgl. Auer 2000). Im Italienischen betrachten wir nachgestellte sachverhaltsbezogene Konditionalsätze wie in (114) als VP-Adjunkte:

(109) Er kann, wenn er nicht müde ist, gut Treppen steigen. (linkes IP-Adjunkt)

(110) Dieser Schutz wird für maximal zwei Jahre gewährt, aber der Rat kann dem gemeinsam, wenn die Bedingungen gegeben sind, ein Ende setzen. (linkes VPAdjunkt)

\footnotetext{
${ }^{9}$ In die Testbeispiele haben wir zur Verdeutlichung Intonationen eingetragen, mit denen sie zu realisieren wären, wenn die Konditionalsätze abgespalten sind. Die gleichen Wortketten würden mit anderen Intonationen auch Lesarten erlauben, bei denen es sich nicht um Spaltsätze handelt und die nicht sinnlos oder unplausibel sind.
} 
(111) Wir freuen uns, wenn uns die Kommissarin über den gegenwärtigen Stand der Dinge informiert. (rechtes IP-Adjunkt)

(112) Se le condizioni lo consentono, gli agricoltori producono in quantità sufficiente. (linkes IP-Adjunkt)

Wenn die Gegebenheiten es zulassen, produzieren die Landwirte ausreichend.

(113) Anche Marta, se sua mamma starà meglio, verrà con noi in vacanza. (linkes VPAdjunkt)

Auch Marta, wenn es ihrer Mutter besser geht, fährt mit uns in Urlaub.

(114) Non ci saranno altri ostacoli in futuro, se i paesi ricchi metteranno a disposizione fondi sufficienti. (rechtes VP-Adjunkt)

In Zukunft werden keine anderen Hindernisse bestehen, wenn die reichen Länder genügend Mittel zur Verfügung stellen.

Wahrheitsbezogene Konditionalsätze können ebenfalls alle Integrationspositionen einnehmen. Als linke Adjunkte zur VP wie in (116) und (119) haben sie Parenthese-Charakter. Auch in (115) ist eine Analyse des wenn-Satzes als VP-Adjunkt möglich; dann ergibt sich nach unserem Eindruck ebenfalls ein Parenthese-Effekt. Im Italienischen analysieren wir nachgestellte wahrheitsbezogene Konditionalsätze wie in (120) als IP-Adjunkte:

(115) Er hat, wenn ich recht verstanden habe, gestern wegen Gesundheitsproblemen abgesagt. (linkes IP-Adjunkt)

(116) Ich habe so etwas, wenn ich mich nicht irre, schon gehört. (linkes VP-Adjunkt)

(117) Das meiste hast du ja schon erledigt, wenn ich richtig gelesen habe. (rechtes IPAdjunkt)

(118) Se non mi sbaglio, lei ha detto che il termine di presentazione è fissato per mercoledì 3 luglio. (linkes IP-Adjunkt)

Wenn ich mich nicht irre, hat sie gesagt, dass die Abgabefrist auf Mittwoch, den 3. Juli, festgelegt wurde.

(119) Anche lui, se ho capito bene, è d'accordo con la nostra proposta. (linkes VPAdjunkt)

Auch er, wenn ich richtig verstanden habe, ist mit unserem Vorschlag einverstanden.

(120) La Commissione ci dice che sta attendendo che il governo algerino voglia negoziare, se ho letto bene. (rechtes IP-Adjunkt)

Die Kommission sagt uns, sie warte darauf, daß die algerische Regierung verhandeln will, wenn ich richtig gelesen habe.

Sprechaktbezogene Konditionalsätze haben in allen Positionen im Satzinneren ParentheseCharakter (vgl. d'Avis 2004: 144):

(121) Auch ich kann, wenn Sie mir die Bemerkung gestatten, unmöglich verstehen, wie Sie mit Ihrem Äußeren zufrieden sein können. (linkes VP-Adjunkt) 
(122) Tutti insieme, se così si può dire, daremo alla luce l'Europa ideale. (linkes VPAdjunkt)

Alle zusammen, wenn man so sagen darf, verwirklichen wir das ideale Europa.

Oft werden sie durch Gedankenstriche oder ähnliche Interpunktionsmittel (bzw. prosodische Mittel) abgegrenzt, die als Desintegrationsmarker zu deuten sind (vgl. Günthner 1999b: 221f.). (123) und (124) sind Beispiele für sprechaktbezogene Konditionalsätze in der REAPosition. Wenn nachgestellte sachverhalts- oder wahrheitsbezogene Konditionalsätze interpunktorisch oder prosodisch abgegrenzt werden, sind sie als Nachträge zu lesen:

(123) Kein Psalm führt uns auf die Höhe des Epheser-Briefes, und doch sind sie auf uns zugeschnitten - wenn man so sagen darf.

(124) In Giappone i prezzi sono di gran lunga inferiori ai nostri - se me lo chiedi. In Japan sind die Preise viel niedriger als bei uns - wenn du mich fragst.

Sprechaktbezogene Konditionalsätze sind auch in der LEA-Position häufig:

(125) Wenn ich fragen darf: Als was arbeitest du jetzt?

(126) Se devo proprio rispondere alla tua domanda: è meglio un iMac anche usato che un Macmini.

Wenn ich wirklich auf deine Frage antworten soll: Auch ein gebrauchter iMac ist besser als ein Macmini.

Wahrheitsbezogene Konditionalsätze in LEA-Stellung kommen ebenfalls vor:

(127) Wenn ich es mir genau überlege: Deine Aussage macht eventuell Sinn.

(128) Se mi ricordo bene: ieri siamo arrivati al secondo capitolo.

Wenn ich mich richtig erinnere: Gestern sind wir bis zum zweiten Kapitel gekommen.

Dagegen sind sachverhaltsbezogene Konditionalsätze in LEA-Position in beiden Sprachen marginal (zum Deutschen vgl. Günthner 1999b).

Als Nacherst-Parenthesen kommen Konditionalsätze aller drei Arten vor, sachverhaltsbezogene aber deutlich seltener als wahrheits- und sprechaktbezogene:

(129) Meine Lehrerin, wenn sie etwas falsch macht, sagt immer: "Das habe ich doch absichtlich gemacht!" (sachverhaltsbezogen)

(130) Con mia sorella, se torna a casa questo fine settimana, dipingo tutta la mia stanza. (sachverhaltsbezogen)

Mit meiner Schwester, wenn sie dieses Wochenende zurückkommt, bemale ich mein ganzes Zimmer.

(131) Dein Vater (wenn ich dich richtig verstanden habe) hat die Fondsanteile geerbt. (wahrheitsbezogen)

(132) Quella foto, se posso dirvelo, l'hanno già inviata al concorso della National Geographic. (sprechaktbezogen)

Dieses Bild, wenn ich es euch sagen darf, haben sie schon beim Wettbewerb von National Geographic eingereicht. 


\subsection{Obwohl- und sebbene-Sätze}

Obwohl und sebbene leiten konzessive Adverbialsätze ein (vgl. Di Meola 1997: 168-232, 275-314; Fahrländer 2013; Renzi/Salvi/Cardinaletti 2001, Bd. 2: 809-817). Neben seiner Verwendung als Subjunktor kann obwohl auch als Konjunktor verwendet werden, vor allem in informeller Rede (vgl. Günthner 1996; Günthner 1999a; Fahrländer 2013: 8f.). Obwohl als Konjunktor steht in der Nullposition seines zweiten Konnekts. Ein Beispiel aus Breindl (2004: 27):

(133) Nein danke, ich will keine Kekse mehr, obwohl: ich nehm noch einen.

Auch sebbene kann (informell) als Konjunktor gebraucht werden. Das erste Beispiel stammt aus Giacoma / Kolb (2001: 2160), das zweite aus dem Internet:

(134) È ancora presto, sebbene, faresti meglio a incamminarti.

Es ist noch früh, aber es wäre besser, wenn du jetzt aufbrechen würdest.

(135) Affinché io non vada avanti ridendo così, come colui il quale dice sciocchezze, sebbene che cosa vieta che uno che ride dica il vero?

Damit ich nicht so lachend weiterspreche wie einer, der Schwachsinn redet, obwohl - was spricht dagegen, dass einer, der lacht, die Wahrheit sagt?

Obwohl und sebbene können wie wenn und se auf der Sachverhaltsebene, der epistemischen Ebene und der illokutionären Ebene verknüpfen. Obwohl-Sätze aller drei Lesarten können im Vorfeld ihres Matrixsatzes stehen:

(136) Obwohl Berlin sich ständig verändert, sind viele Zeugnisse aus der Vergangenheit heute noch erlebbar. (sachverhaltsbezogen)

(137) Obwohl es nicht so aussieht, könnte es sein, dass das Gerät kaputt ist. (epistemisch)

(138) Obwohl du es schon weißt, möchte ich noch einmal erwähnen, dass alle Produkte direkt aus Italien stammen. (sprechaktbezogen)

Für sprechaktbezogene Verknüpfungen wie (138) ist desintegrierte LEA-Stellung typischer:

(139) Obwohl du es schon weißt: Ich habe eine dreistellige Zahl an Rehen mit der 9,3 und dem $17 \mathrm{~g}$ StaM geschossen.

Drei Beispiele für sebbene-Sätze in SpC-Position:

(140) Sebbene nessuno a casa sia d'accordo, la macchina la venderò prima delle vacanze. (sachverhaltsbezogen)

wörtl.: Obwohl niemand bei mir zu Hause damit einverstanden ist, das Auto das verkaufe ich vor den Ferien.

(141) Sebbene non sembri così, la casa l'ho recentemente restaurata. (epistemisch) wörtl.: Obwohl es nicht so aussieht, das Haus das habe ich vor kurzem renoviert.

(142) Sebbene tu lo sappia già, il buio devi affrontarlo da solo. (sprechaktbezogen) wörtl.: Obwohl du es schon wusstest, die Dunkelheit die musst du allein ertragen.

In illokutionären Verknüpfungen wie (142) kann der vorangestellte sebbene-Satz auch als desintegriert (LEA-Position) gedeutet werden, insbesondere wenn anstelle des Kommas ein Doppelpunkt gesetzt wird. 
Obwohl- und sebbene-Sätze sind kaum abspaltbar und bestehen auch die übrigen Tests für starke Integration (Negierbarkeit, Erfragbarkeit, Korrelatfähigkeit usw.; s.o. Abschnitt 2.1) nicht. Möglicherweise kann daraus geschlossen werden, dass sie nur schwach integriert und desintegriert verwendet werden können. Zifonun et al. (1997: 2314f.) weisen allerdings darauf hin, dass mangelnde Abspaltbarkeit usw. bei Konzessivsätzen möglicherweise nicht syntaktisch, sondern semantisch bedingt sind (vgl. Blühdorn 2008a: 230f.). Diese Frage können wir hier nicht abschließend klären.

Obwohl- und sebbene-Sätze können als linke IP- und VP-Adjunkte sowie als rechte IPAdjunkte auftreten. Im Satzinneren haben sie in allen Lesarten Parenthese-Charakter wie in den Beispielen (143), (144), (145) und (149):

(143) Sie hat, obwohl die Bühne sehr klein war, eine super Show gemacht. (linkes IPAdjunkt; sachverhaltsbezogen)

(144) Frau Müller scheint, obwohl sie nicht sehr gesprächig ist, ein gutes Herz zu haben. (linkes IP-Adjunkt; epistemisch)

(145) Ich nehme den Wagen, obwohl er als 3-Türer besser aussieht, als 5-Türer, da wir zwei Kinder haben. (sachverhaltsbezogen; linkes VP-Adjunkt)

(146) Deine Eltern machen sich wahrscheinlich Sorgen um dich, obwohl du es nicht glauben wirst. (rechtes IP-Adjunkt; sprechaktbezogen)

(147) Sebbene i pescatori ne siano stati avvertiti, le difficoltà non sono diminuite. (linkes IP-Adjunkt; sachverhaltsbezogen)

Obwohl die Fischer gewarnt wurden, haben sich die Schwierigkeiten nicht vermindert.

(148) Sebbene la mia opinione non valga nulla, io ritengo che il giornale non si vende più perché è un prodotto scadente. (linkes IP-Adjunkt; sprechaktbezogen)

Obwohl meine Meinung nicht zählt, glaube ich, dass die Zeitung sich deshalb nicht besser verkauft, weil sie schlecht gemacht ist.

(149) Paolo, sebbene non lo dimostri, ha sicuramente piacere ad incontrarci. (linkes VPAdjunkt; epistemisch)

Paolo, obwohl er es nicht zeigt, freut sich sicherlich darauf, uns zu treffen.

(150) L'Italia è tecnicamente fallita da anni, sebbene non vi interessi la cosa. (rechtes IPAdjunkt; sprechaktbezogen)

Italien ist technisch seit Jahren pleite, obwohl euch die Sache nicht interessiert.

In Beispiel (148) kann der vorangestellte, in (146) und (150) der nachgestellte obwohl- bzw. sebbene-Satz auch als desintegriert (LEA- bzw. REA-Position) analysiert werden. Diese Deutungen würden durch die Setzung eines Doppelpunktes bzw. Gedankenstrichs gefördert.

Auch sachverhaltsbezogene und epistemische obwohl- und sebbene-Sätze können die LEAoder REA-Position einnehmen. Bei Sachverhaltsbezug ist LEA-Stellung aber nur in informeller Rede $\mathrm{zu}$ erwarten; REA-Stellung ist typisch für Nachträge. Als NacherstParenthesen sind obwohl- und sebbene-Sätze in allen Lesarten häufig: 
(151) Auch wir, obwohl wir als Erstlingsgabe den Geist haben, seufzen in unserem Herzen. (sachverhaltsbezogen)

(152) Sie (obwohl du es weißt) hat einen Freund. (sprechaktbezogen)

(153) Per mio figlio, sebbene non se lo meriti, cerco sempre il meglio. (sachverhaltsbezogen)

Für meinen Sohn, obwohl er es nicht verdient, suche ich immer das Beste.

(154) Questa versione dei fatti (sebbene tu non lo sappia ancora) me l'ha già raccontata Gianni ieri. (sprechaktbezogen)

Diese Version der Geschichte (obwohl du es noch nicht weift) hat mir Gianni schon gestern erzählt.

\subsection{Weil- und perché-Sätze}

Weil und perché leiten kausale Adverbialsätze ein (vgl. Ravetto/Blühdorn 2011). Ebenso wie obwohl und sebbene kann weil neben seiner Verwendung als Subjunktor in informeller Rede als Konjunktor dienen (vgl. Günthner 1996; Uhmann 1998; Freywald 2009: 117; Antomo/Steinbach 2010; Volodina 2011: 74-87). In dieser Funktion kann es - ebenso wie standardsprachlich denn - V1- oder V2-Sätze einleiten (vgl. Blühdorn 2008c: 5):

(155) Wo sind die Killmails von 2003 bis 2005 geblieben? Weil haben sie nicht erst 2008 oder 2009 eingeführt, dass man keine Killmails mehr bekommt? (V1-Satz)

(156) Ich darf nicht böse mit der Redakteurin werden, weil ich bin auf sie angewiesen. (V2-Satz)

Perché-Sätze werden in der weit überwiegenden Mehrzahl der Fälle nachgestellt, sind aber bei Voranstellung nicht ungrammatisch (vgl. Previtera 1996: 35; Ferraris 1999: 68; Lombardi Vallauri 2000: 65f.; Serianni 2000: 401; Ravetto/Blühdorn 2011: 218-228). Perché ist nicht als Konjunktor zu betrachten, da perché-Verknüpfungen stets das subordinationstypische Bindungsverhalten zeigen (vgl. Renzi/Salvi/Cardinaletti 2001, Bd. 2: 749). Variable in koordinierten weil-Sätzen können nicht durch Quantoren im anderen Konnekt gebunden werden (vgl. Antomo/Steinbach 2010: 11f.), Variable in perché-Sätzen dagegen ohne weiteres:

$(157) *$ Niemandi war verärgert, weil eri wurde nicht eingeladen.

(158) Nessuno $_{i}$ se l'è presa, perché $\emptyset_{i}$ non è stato invitato.

Auch weil und perché können auf der Sachverhaltsebene, der epistemischen Ebene und der illokutionären Ebene verknüpfen (vgl. Sweetser 1990: 76-86; Blühdorn 2006: 265-268; Antomo/Steinbach 2010: 17f.; Ravetto/Blühdorn 2011: 228-235). Sachverhaltsbezogene weil-Sätze können im Vorfeld ihres Matrixsatzes stehen:

(159) Weil ich nicht bezahlt habe, wurde mein Konto gesperrt.

Epistemische und sprechaktbezogene weil-Sätze sind in dieser Position nicht zugelassen (vgl. Konopka 2006: 110; Blühdorn 2006: 276; Blühdorn 2008a: 222f.; Ravetto/Blühdorn 2011: 237). Sachverhaltsbezogene perché-Sätze sind gelegentlich in SpC-Position zu finden: 
(160) Perché non avevo alternative, suo figlio l'ho fatto venire a casa mia. wörtl.: Weil ich keine andere Möglichkeit hatte, seinen Sohn den habe ich bei mir aufgenommen.

Für vorangestellte epistemische und illokutionäre perché-Sätze scheint uns die Annahme, dass sie eine SpC-Position einnehmen können, nicht gerechtfertigt. Epistemische perchéSätze wie in (161) können eventuell linke IP-Adjunkte sein. Am plausibelsten ist unseres Erachtens für Fälle wie (161) und (162) die Annahme, dass der perché-Satz in der LEA-Position steht:

(161) Perché contiene sostanze cancerogene, il prodotto non è sicuramente in vendita. (epistemisch)

Da es Krebs erregende Stoffe enthält, ist das Produkt sicherlich nicht im Handel.

(162) Proprio perché insisti, la giusta interpretazione la trovi nelle ultime righe dell'Antonini. (sprechaktbezogen)

Da du darauf bestehst, die richtige Interpretation findest du in den letzten Zeilen von Antonini.

Nur für sachverhaltsbezogene weil- und perché-Sätze ist anzunehmen, dass sie syntaktisch integriert sind. Da sie zugleich abgespalten werden können, müssen sie stark integriert sein:

(159a) Es ist, weil ich nicht bezahlt habe, dass mein Konto gesperrt wurde.

(160a) Era perché non avevo alternative, che ho fatto venire suo figlio a casa mia.

Sachverhaltsbezogene weil- und perché-Sätze können alle Integrationspositionen einnehmen:

(163) Man hat, weil keine überflüssige Kochstelle an Bord ist, in der Breite sehr viel Platz. (linkes IP-Adjunkt)

(164) Ich habe meine Tochter, weil sie morgen eine Klausur schreibt, extra früh ins Bett geschickt. (linkes VP-Adjunkt)

(165) Ich bin total deprimiert, weil ich zunehme. (rechtes IP-Adjunkt)

(166) Perché ha bisogno di mangiare, lui ruba ogni giorno in un supermercato diverso. (linkes IP-Adjunkt)

Weil er etwas zum Essen braucht, klaut er jeden Tag in einem anderen Supermarkt.

(167) Mia madre, perché ha sempre tante cose da fare, qualche volta si dimentica che esisto. (linkes VP-Adjunkt)

Meine Mutter, weil sie immer so viel zu tun hat, vergisst manchmal, dass ich existiere.

(168) 300 pescatori mauritani hanno perso la vita lo scorso anno, perché le condizioni di cattura sono diventate più pericolose. (rechtes VP-Adjunkt)

300 mauretanische Fischer haben im vergangenen Jahr ihr Leben verloren, weil die Fangbedingungen gefährlicher geworden sind.

Epistemische und illokutionäre weil-Sätze haben im Satzinneren, soweit sie dort vorkommen, stets Parenthese-Charakter. Meist wird für solche Funktionen da als kausaler Subjunktor bevorzugt: 
(169) Ich sagte, weil du mich fragst, nein! (linkes IP-Adjunkt; bevorzugt: ...da $d u$ mich fragst...)

Epistemische und illokutionäre perché-Sätze kommen im Satzinneren häufiger vor. Sie sind dort ebenfalls stets Parenthesen:

(170) Io, proprio perché me lo chiedi, non ho intenzione di rinunciare al mio lavoro. (linkes VP-Adjunkt)

Ich, da du mich danach fragst, habe nicht die Absicht, auf meinen Job zu verzichten.

Nachgestellte epistemische und illokutionäre weil-Verbletztsätze sind syntaktisch desintegriert (REA-Position). Oft, aber nicht immer, werden sie durch die Interpunktion bzw. Prosodie entsprechend gekennzeichnet (vgl. Breindl/Walter 2009: 56f.):

(171) Die meisten Anwender machen bestimmt mit, weil sie ja nichts zu verbergen haben. (epistemisch)

(172) Ich persönlich habe nichts dagegen, wenn meine Freundin in die Sauna geht - weil du mich fragst. (illokutionär)

Nachgestellte epistemische und illokutionäre perché-Sätze sind ebenfalls als desintegriert zu betrachten:

(173) Vero, c'è qualcosa che mi ronza nella testa dalla sera del ballo - solo perché me lo chiedi. (illokutionär)

Richtig, es gibt etwas, was mir seit dem Abend des Tanzes durch den Kopf geht-nur weil du mich danach fragst.

Als Nacherst-Parenthesen können weil- und perché-Sätze aller drei Gruppen auftreten. Für sachverhaltsbezogene Sätze ist diese Position weniger typisch als für epistemische und illokutionäre:

(174) Die Entscheidung, weil sie schwierig ist, trifft die Mama. (sachverhaltsbezogen)

(175) A Roberto, perché è scomparso senza dire nulla, sarà probabilmente venuto un grosso senso di colpa. (epistemisch)

Robert, weil er verschwunden ist, ohne etwas zu sagen, wird wahrscheinlich das schlechte Gewissen quälen.

(176) Ich selbst - weil du mich fragst - habe an allen Autos (Ausnahme: Porsche) Interesse. (sprechaktbezogen)

\section{$5 \quad$ Fazit}

Wir haben in diesem Aufsatz die unterschiedlichen Grade syntaktischer Integration von adverbialen Nebensätzen im Deutschen und Italienischen untersucht. Dazu haben wir in einem ersten Schritt drei Klassen von Adverbialia unterschieden:

(i) Stark integrierte (zentrale) Adverbialia können eine SpC-Position einnehmen und können abgespalten werden.

(ii) Schwach integrierte (periphere) Adverbialia können eine SpC-Position einnehmen, aber nicht abgespalten werden. 
(iii) Desintegrierte Adverbialia können keine SpC-Position einnehmen und auch nicht abgespalten werden.

Anschließend haben wir acht Klassen von Positionen in der Linearstruktur des Satzes unterschieden, die in beiden Vergleichssprachen mit Adverbialia besetzt werden können:

(a) Integrationspositionen: SpC-Positionen sowie die linken und rechten Adjunktpositionen zur VP und zur IP.

(b) Desintegrationspositionen: LEA- und REA-Position sowie die Nacherststelle.

Im dritten Schritt haben wir für zehn Klassen von Subjunktorsätzen, fünf aus dem Deutschen und fünf aus dem Italienischen, aufgrund empirischer Daten die Verteilung auf diese Linearpositionen untersucht, wobei wir auch unterschiedliche Verknüpfungslesarten berücksichtigt haben. Abbildung 5 gibt einen Überblick über die gefundenen Verteilungen.

\begin{tabular}{|l|c|c|c|c|c|c|c|c|c|}
\cline { 2 - 10 } \multicolumn{1}{c|}{} & LEA & SpC & NE & liIP & liVP & rVP & rIP & REA & NS \\
\hline als & $(\mathrm{t})$ & $\mathrm{t}$ & $([\mathrm{t}])$ & $\mathrm{t}$ & $\mathrm{t}$ & - & $\mathrm{t}$ & $\mathrm{t} / \mathrm{w}$ & - \\
\hline quando & $\mathrm{t})$ & $\mathrm{t}$ & $([\mathrm{t}])$ & $\mathrm{t}$ & $\mathrm{t}$ & $\mathrm{t}$ & - & $\mathrm{t} / \mathrm{w}$ & - \\
\hline während & $\mathrm{t}) / \mathrm{a}$ & $\mathrm{t} / \mathrm{a}$ & $([\mathrm{t}] /[\mathrm{a}])$ & $\mathrm{t} /(\mathrm{a})$ & $\mathrm{t} /[\mathrm{a}]$ & - & $\mathrm{t} / \mathrm{a}$ & $\mathrm{t} / \mathrm{a}$ & - \\
\hline mentre & $(\mathrm{t}) / \mathrm{a}$ & $\mathrm{t} / \mathrm{a}$ & {$[\mathrm{t}] /[\mathrm{a}]$} & $\mathrm{t} / \mathrm{a}$ & $\mathrm{t} /[\mathrm{a}]$ & $\mathrm{t}$ & $\mathrm{a}$ & $\mathrm{t} / \mathrm{a}$ & - \\
\hline wenn & $(\mathrm{s}) / \mathrm{e} / \mathrm{i}$ & $\mathrm{s} / \mathrm{e} /(\mathrm{i})$ & $([\mathrm{s}]) /[\mathrm{e}] /[\mathrm{i}]$ & $\mathrm{s} /[\mathrm{e}] /[\mathrm{i}]$ & $\mathrm{s} /[\mathrm{e}] /[\mathrm{i}]$ & - & $\mathrm{s} / \mathrm{e} /(\mathrm{i})$ & $\mathrm{s} / \mathrm{e} / \mathrm{i}$ & - \\
\hline se & $(\mathrm{s}) / \mathrm{e} / \mathrm{i}$ & $\mathrm{s} / \mathrm{e} /(\mathrm{i})$ & $([\mathrm{s}]) /[\mathrm{e}] /[\mathrm{i}]$ & $\mathrm{s} / \mathrm{e} / \mathrm{i}$ & $\mathrm{s} /[\mathrm{e}] /[\mathrm{i}]$ & $\mathrm{s}$ & $\mathrm{e} /(\mathrm{i})$ & $\mathrm{s} / \mathrm{e} / \mathrm{i}$ & - \\
\hline obwohl & $(\mathrm{s}) / \mathrm{e} / \mathrm{i}$ & $\mathrm{s} / \mathrm{e} /(\mathrm{i})$ & {$[\mathrm{s}] /[\mathrm{e}] /[\mathrm{i}]$} & {$[\mathrm{s}] /[\mathrm{e}] /[\mathrm{i}]$} & {$[\mathrm{s}] /[\mathrm{e}] /[\mathrm{i}]$} & - & $\mathrm{s} / \mathrm{e} /(\mathrm{i})$ & $\mathrm{s} / \mathrm{e} / \mathrm{i}$ & $(\mathrm{K})$ \\
\hline sebbene & $(\mathrm{s}) / \mathrm{e} / \mathrm{i}$ & $\mathrm{s} / \mathrm{e} /(\mathrm{i})$ & {$[\mathrm{s}] /[\mathrm{e}] /[\mathrm{i}]$} & $\mathrm{s} / \mathrm{e} / \mathrm{i}$ & {$[\mathrm{s}] /[\mathrm{e}] /[\mathrm{i}]$} & - & $\mathrm{s} / \mathrm{e} /(\mathrm{i})$ & $\mathrm{s} / \mathrm{e} / \mathrm{i}$ & $\mathrm{K}$ \\
\hline weil & $(\mathrm{e} / \mathrm{i})$ & $\mathrm{s}$ & $([\mathrm{s}]) /[\mathrm{e}] /[\mathrm{i}]$ & $\mathrm{s} /([\mathrm{e}] /[\mathrm{i}])$ & $\mathrm{s} /([\mathrm{e}] /[\mathrm{i}])$ & - & $\mathrm{s}$ & $\mathrm{s} / \mathrm{e} / \mathrm{i}$ & $(\mathrm{K})$ \\
\hline perché & $(\mathrm{e} / \mathrm{i})$ & $(\mathrm{s})$ & $([\mathrm{s}] /[\mathrm{e}] /[\mathrm{i}]$ & $\mathrm{s} /(\mathrm{e} / \mathrm{i})$ & $\mathrm{s} /[\mathrm{e}] /[\mathrm{i}]$ & $\mathrm{s}$ & - & $\mathrm{s} / \mathrm{e} / \mathrm{i}$ & - \\
\hline
\end{tabular}

Abb. 5: Verteilungsprofile der untersuchten Subjunktorsätze

Legende: LEA - linke externe Adjunktposition; SpC - Spezifizierer einer C-Projektion; NE Nacherstposition; liIP, liVP, rVP, rIP - linkes bzw. rechtes IP- bzw. VP-Adjunkt; REA rechte externe Adjunktposition; NS - nächster Satz; $\mathrm{t}$ - temporal; w - weiterführend; $\mathrm{a}$ - adversativ; s - sachverhaltsbezogen; e - epistemisch; i - illokutionär; K - Verwendung als Konjunktor; runde Klammern - seltenes Vorkommen und/oder Beschränkung auf informelle Rede; eckige Klammern - Parenthese-Charakter.

Die wichtigste Verallgemeinerung lautet: Integrierte Adverbialsätze bevorzugen Integrationspositionen, desintegrierte bevorzugen Desintegrationspositionen. Daraus ergibt sich allerdings für keine der untersuchten Adverbialsatzklassen eine ausschließliche Festlegung. Im Gegenteil: Adverbialsätze aller Klassen können unterschiedliche Linearpositionen einnehmen, und zwar sowohl Integrations- als auch Desintegrationspositionen. Deshalb lohnt es sich, die Verteilung im Detail anzuschauen:

- Stark integrierte Adverbialsätze (temporale als-, quando-, während- und mentreSätze sowie sachverhaltsbezogene wenn-, se-, weil- und perché-Sätze) bevorzugen Adjunktpositionen zur VP oder IP oder SpC-Positionen. Bei Nachstellung haben wir sie im Italienischen als VP-Adjunkte, im Deutschen als IP-Adjunkte analysiert. LEA-Stellung entspricht für stark integrierte Adverbialsätze nicht dem Standard. In 
informeller Rede kommt sie aber vor. REA-Stellung ist möglich und führt zu weiterführenden oder Nachtrags-Lesarten. Parenthetische Nacherststellung ist möglich, aber untypisch.

- Schwach integrierte Adverbialsätze (adversative während- und mentre-Sätze, epistemische wenn- und se-Sätze sowie sachverhaltsbezogene und epistemische obwohlund sebbene-Sätze) bevorzugen die rechte Adjunktposition zur IP oder SpCPositionen. Die linke Adjunktposition zur VP oder zur IP können sie ebenfalls einnehmen. Im Satzinneren bekommen sie dann Parenthese-Charakter. Ferner können sie ohne Lesartveränderung die LEA- oder die REA-Position einnehmen. Parenthetische Nacherststellung ist möglich, für während-Sätze aber untypisch.

- Desintegrierte Adverbialsätze bevorzugen Stellungen am Satzrand oder als Nacherst-Parenthesen. Zwei Untergruppen sind zu unterscheiden:

- Sprechaktbezogene wenn-, se-, obwohl- und sebbene-Sätze können Desintegrationspositionen, aber auch Integrationspositionen am Satzrand einnehmen, also die LEA- oder die REA-Position, im Italienischen die linke IP-Adjunkt-Position, in beiden Sprachen (seltener) die rechte IP-Adjunkt-Position und auch SpCPositionen. In Integrationspositionen im Satzinneren (als linkes IP-Adjunkt im Deutschen oder als linkes VP-Adjunkt in beiden Sprachen) kommen sie ebenfalls vor, aber stets nur als Parenthesen.

- Epistemische und sprechaktbezogene weil- und perché-Sätze bevorzugen Desintegrationspositionen am Satzrand, insbesondere die REA-Position. Stellung in Integrationspositionen im Satzinneren ist möglich, aber nur als Parenthese. LEA-Stellung ist möglich, aber selten; für weil-Sätze entspricht sie nicht dem Standard. Für perché-Sätze sind Verwendungen als linkes IP-Adjunkt nicht auszuschließen.

Es gibt weitere Unterklassen desintegrierter Adverbialsätze, etwa Postponierersätze, die nur in der REA-Position zugelassen sind (s. o. Kapitel 2). Die Skala der syntaktischen Integrationsund Subordinationsgrade ist also empirisch in mehr als drei Stufen aufgefächert.

Die beobachtete Verteilung zeigt große Ähnlichkeiten zwischen den Subjunktorpaaren aus den Vergleichssprachen, bei denen es sich um partielle Bedeutungsäquivalente handelt. Wenn- und se-Sätze zeigen in sachverhaltsbezogener, epistemischer und illokutionärer Verwendung jeweils eine andere syntaktische Verteilung. Bei obwohl- und sebbene-Sätzen verhalten sich sachverhaltsbezogene und epistemische Verwendungen ähnlich und unterscheiden sich von illokutionären Verwendungen. Bei weil- und perché-Sätzen verhalten sich umgekehrt epistemische und illokutionäre Verwendungen ähnlich und unterscheiden sich von sachverhaltsbezogenen.

Die Distributionsunterschiede in der linken IP-Adjunkt-Position und der rechten VP-AdjunktPosition sind durchgängig und auf allgemeine Strukturunterschiede zwischen den Vergleichssprachen zurückzuführen: Im Italienischen liegt die linke IP-Adjunkt-Position typischerweise am Satzanfang, im Deutschen im Satzinneren; die rechte VP-Adjunkt-Position ist im Deutschen für Adverbialsätze nicht zugelassen. 


\section{Nachsatz: Thesen zum Verhältnis zwischen Grammatikforschung und Sprachdaten}

Wir nehmen die detaillierten Kommentare einer LO-GutachterIn über unsere Arbeit mit Belegmaterial zum Anlass, Auskunft über einige methodische Grundgedanken zu geben, denen wir in dieser und anderen Untersuchungen gefolgt sind:

1. Eine realitätsgerechte Grammatikforschung muss sich auf Belege und auf Kompetenzurteile stützen. Beides sind empirische Sprachdaten. Weder das eine noch das andere kann der Grammatikforschung allein als Grundlage genügen. Belege sind nur dann nützlich, wenn die Kompetenz des Forschers mit ihnen in Dialog tritt. Im Zweifelsfall muss der Grammatiker den einzelnen Beleg anschauen, kritisch prüfen und deuten.

2. Die Introspektion des Muttersprachlers hat Zugriff auf einen mehr oder weniger großen Teil der Grammatik. Was in einer Sprache insgesamt möglich ist, kann durch Introspektion aber nicht ermittelt werden. Die Vielfalt der grammatischen Erscheinungen zeigt sich letztlich nur in Datenkorpora. Nur ein gründliches Datenstudium schützt vor einem unrealistisch verengten Bild von der Sprache.

3. Selbst in sehr großen Korpora können Belege für bestimmte Erscheinungen, die die Kompetenz eines Muttersprachlers widerstandslos anerkennt, schwer auffindbar sein, z. B. weil die Erscheinung selten vorkommt oder weil sie nicht leicht zu suchen ist. Andererseits finden sich in jedem umfangreicheren Datenkorpus Belege, die die Kompetenz von Muttersprachlern aus unterschiedlichen Gründen und mit unterschiedlicher Schärfe - zurückweist. Bei solchen Daten kann es sich um Fehler handeln. Ihnen können aber auch Regeln oder Normen zugrundeliegen, die nur zur Kompetenz einer Teilmenge der Sprachteilhaber gehören oder nur für Teilgebiete des Zeichenverkehrs in der Sprachgemeinschaft gelten (bestimmte Textsorten, Themenbereiche, Verkehrsformen usw.). Um solche Daten erkennen und realitätsgerecht beschreiben zu können, werden Kompetenzurteile benötigt. Wenn die Urteilsfähigkeit des Grammatikforschers nicht ausreicht, müssen Informanten befragt werden.

4. Korpusbelege sind für die Arbeit des Grammatikforschers nicht immer nur hilfreich, sondern manchmal durchaus auch störend, z. B. wenn sie neben denjenigen Eigenschaften, die untersucht werden sollen, weitere Eigenschaften aufweisen, die auf schwer abschätzbare Weise mit den untersuchten Eigenschaften interagieren. In solchen Fällen kann es nicht nur legitim, sondern sogar methodisch geboten sein, Korpusbelege zu bearbeiten, z. B. zu kürzen oder zu vereinfachen. Es kann auch sinnvoll sein, Fehler zu korrigieren, die für die aktuell untersuchte Frage keine Rolle spielen, in der Argumentation aber vom Wesentlichen ablenken würden. Auch Beispiele, die der Grammatikforscher in der Muttersprache selbst gebildet hat, können legitime Belegdaten sein, z. B. wenn thematisch geeignete Fremdbelege nicht verfügbar oder durch Urheber- oder Persönlichkeitsrechte geschützt sind. Beispiele sollen aber nach Möglichkeit nicht "frei" erfunden werden, sondern sich eng an vorhandenen Korpusdaten orientieren, um Selbsttäuschungen zu vermeiden.

5. Für die Bearbeitung vieler Fragestellungen der Grammatikforschung erscheint uns das Internet als eine attraktive Belegquelle, die Vorteile vor anderen Belegquellen hat. Seine größte Stärke ist sein Umfang. Kein anderes Datenkorpus kann die schriftlich realisierbaren Erscheinungsformen einer Sprache in ähnlicher Bandbreite und Aktualität abbilden. Außerdem enthält das Internet vergleichbare Daten aus zahlreichen Sprachen, was in dieser Form erst recht kein anderes Korpus bieten kann. Hinzu kommen praktische Vorzüge wie die leichte, infor- 
melle Bedienbarkeit über Suchmaschinen, die freie, ortsunabhängige Zugänglichkeit und die Reproduzierbarkeit von Suchvorgängen und Daten. Belege können mit minimalem Aufwand wiedergefunden werden, und wo dies nicht möglich sind, kann leicht nach analogen Beispielen gesucht werden. Für die Fragestellungen, die wir in diesem und anderen Aufsätzen bearbeitet haben, haben die genannten Vorzüge den Ausschlag gegeben, das Internet als Datenbasis zu wählen. Dass für Untersuchungen anderer Fragestellungen möglicherweise andere Datenkorpora zu bevorzugen sind, wird damit keineswegs bestritten.

6. Transparenz, Explizitheit und Nachvollziehbarkeit sind unverzichtbare Eigenschaften einer seriösen wissenschaftlichen Arbeit. Ihre Verabsolutierung kann allerdings zu weltfremden Fiktionen führen. Der Grammatikforscher macht im Rahmen seiner Möglichkeiten immer nur Beschreibungs- und Erklärungsangebote, deren Plausibilität der Leser kritisch prüfen muss. Keine wissenschaftliche Arbeit ist vor Irrtümern gefeit.

\section{Literatur}

Altmann, Hans (1981): Formen der "Herausstellung" im Deutschen. Rechtsversetzung, Linksversetzung, Freies Thema und verwandte Konstruktionen. Tübingen: Niemeyer.

Antomo, Mailin/Steinbach, Markus (2010): "Desintegration und Integration: Weil-V2-Sätze an der Schnittstelle zwischen Syntax, Semantik und Pragmatik". Zeitschrift für Sprachwissenschaft 29/1: 1-37.

Auer, Peter (2000): "Pre- and post-positioning of wenn-clauses in spoken and written German". In: Couper-Kuhlen, Elizabeth/Kortmann, Bernd (eds.) (2000): Cause Condition - Concession - Contrast. Cognitive and Discourse Perspectives. Berlin/New York, de Gruyter: 173-204.

Axel-Tober, Katrin (2012): (Nicht-)kanonische Nebensätze im Deutschen. Synchrone und diachrone Aspekte. Berlin/Boston: de Gruyter.

Benincà, Paola (2001): "The position of Topic and Focus in the left periphery". In: Cinque, Guglielmo/Salvi, Giampaolo (eds.) (2001): Current Studies in Italian Syntax. Essays offered to Lorenzo Renzi. Amsterdam, Elsevier: 39-64.

Bianco, Francesco (2008): Le proposizioni temporali di contemporaneità nella prosa media. http://www.francescobianco.net/linguistica/basel.htm, Stand: 3. Juli 2014.

Blühdorn, Hardarik (2003): "Zur Semantik der Konjunktion als. Paradigmatische und syntagmatische Aspekte". In: Hentschel, Elke (ed.) (2003): Particulae Collectae. Festschrift für Harald Weydt zum 65. Geburtstag. Linguistik online 13: 11-53. https://bop.unibe.ch/linguistik-online/article/view/869/1512, Stand: 3. Juli 2014.

Blühdorn, Hardarik (2006): "Kausale Satzverknüpfungen im Deutschen". Pandaemonium Germanicum 10: 253-282. http://www.revistas.usp.br/pg/article/view/74487/78104, Stand: 3. Juli 2014.

Blühdorn, Hardarik (2008a): "Epistemische Lesarten von Satzkonnektoren - Wie sie zustande kommen und wie man sie erkennt". In: Pohl, Inge (ed.) (2008): Semantik und PragmatikSchnittstellen. Frankfurt/Main, Lang: 217-251.

Blühdorn, Hardarik (2008b): "Subordination and coordination in syntax, semantics and discourse. Evidence from the study of connectives". In: Fabricius-Hansen/Ramm (eds.) (2008): 'Subordination' versus 'Coordination' in Sentence and Text. A cross-linguistic perspective. Amsterdam/Philadelphia, Benjamins: 59-85. 
Blühdorn, Hardarik (2008c): Syntax und Semantik der Konnektoren. Ein Überblick. http://www1.ids-mannheim.de/fileadmin/gra/texte/blu_ueberblick.pdf, Stand: 3. Juli 2014.

Blühdorn, Hardarik (2011): "Informationsstrukturelle Gestaltung von Satzverknüpfungen: Wie interagieren Konnektoren und Prosodie?" In: Breindl, Eva/Ferraresi, Gisella/Volodina, Anna (eds.) (2011): Satzverknüpfungen. Zur Interaktion von Form, Bedeutung und Diskursfunktion. Berlin, de Gruyter: 263-295.

Blühdorn, Hardarik (2012a): "Verknüpfungseigenschaften von Satzkonnektoren im Deutschen. Am Beispiel der Kausal- und Konsekutivkonnektoren". Deutsche Sprache 40: 193-220.

Blühdorn, Hardarik (2012b): Negation im Deutschen. Syntax, Informationsstruktur, Semantik. Tübingen: Narr.

Blühdorn, Hardarik/Lohnstein, Horst (2012): "Verumfokus im Deutschen: Versuch einer Synthese". In: Lohnstein, Horst/Blühdorn, Hardarik (eds.) (2012): Wahrheit - Fokus Negation. Hamburg, Buske: 171-262.

Blühdorn, Hardarik/Ravetto, Miriam (2012): "Die Subjunktoren während und mentre". In: Cinato, Lucia et al. (eds.) (2012): Intrecci di lingua e cultura. Studi in onore di Sandra Bosco Coletsos. Roma, Aracne: 43-64.

Breindl, Eva (2004): "Konzessivität und konzessive Konnektoren im Deutschen". Deutsche Sprache 32: 2-31.

Breindl, Eva/Walter, Maik (2009): Der Ausdruck von Kausalität im Deutschen. Eine korpusbasierte Studie zum Zusammenspiel von Konnektoren, Kontextmerkmalen und Diskursrelationen. Mannheim: Institut für Deutsche Sprache.

Breindl, Eva/Ferraresi, Gisella/Volodina, Anna (eds.) (2011): Satzverknüpfungen. Zur Interaktion von Form, Bedeutung und Diskursfunktion. Berlin: de Gruyter.

Buzzo Margari, Renata (2004): "Considerazioni sulle particelle modali tedesche e sulle corrispondenti espressioni italiane". In: Bosco Coletsos, Sandra/Costa, Marcella (eds.) (2004): Italiano e tedesco: un confronto. Alessandria, Edizioni dell'Orso: 139-171.

Carston, Robyn/Blakemore, Diane (2005): "Introduction to Coordination: Syntax, Semantics and Pragmatics". Lingua 115: 353-358.

Cinque, Guglielmo (1990): Types of A'Dependencies. Cambridge MA: MIT Press.

Cinque, Guglielmo (1999): Adverbs and functional heads: A cross-linguistic perspective. Oxford: Oxford University Press.

Clément, Danièle (1998): "Wie frei sind die Adjunkte? Plädoyer für eine differenzierte syntaktische Beschreibung der Adjunkte am Beispiel der durch während eingeleiteten Adverbialsätze im Deutschen". Deutsche Sprache 26: 38-62.

Coniglio, Marco (2011): Die Syntax der deutschen Modalpartikeln. Ihre Distribution und Lizenzierung in Haupt- und Nebensätzen. Berlin: Akademie Verlag.

Cristofaro, Sonia (2003): Subordination. Oxford: Oxford University Press.

D'Avis, Franz-Josef (2004): "In front of the prefield - inside or outside the clause?" In: Lohnstein, Horst/Trissler, Susanne (eds.) (2004): The Syntax and Semantics of the Left Periphery. Berlin/New York, de Gruyter: 139-177.

Di Meola, Claudio (1997): Der Ausdruck der Konzessivität in der deutschen Gegenwartssprache. Theorie und Beschreibung anhand eines Vergleichs mit dem Italienischen. Tübingen: Niemeyer.

Drach, Erich (1937): Grundgedanken der deutschen Satzlehre. Frankfurt/Main: Diesterweg. 
Ehrich, Veronika et al. (eds.) (2009): Koordination und Subordination im Deutschen. Hamburg: Buske.

Fabricius-Hansen, Cathrine (1992): "Subordination". In: Hoffmann, Ludger (ed.) (1992): Deutsche Syntax. Ansichten und Aussichten. Berlin/New York, de Gruyter: 458-483.

Fabricius-Hansen, Cathrine/Ramm, Wiebke (2008): 'Subordination' versus 'Coordination' in Sentence and Text. A cross-linguistic perspective. Amsterdam/Philadelphia: Benjamins.

Fahrländer, Sarah (2013): Zur Syntax und Semantik der konzessiven Satzkonnektoren obwohl und trotzdem. Mannheim: Institut für Deutsche Sprache (OPAL 1/2013). http://pub.idsmannheim.de/laufend/opal/pdf/opal2013-1.pdf, Stand: 3. Juli 2014.

Ferrari, Angela (2012): Tipi di frase e ordine delle parole. Roma: Carocci.

Ferraris, Stefania (1999): Imparare la sintassi. Vercelli: Mercurio.

Frey, Werner (2011): "Peripheral adverbial clauses, their licensing and the prefield in German". In: Breindl, Eva/Ferraresi, Gisella/Volodina, Anna (eds.) (2011): Satzverknüpfungen. Zur Interaktion von Form, Bedeutung und Diskursfunktion. Berlin, de Gruyter: 41-77.

Freywald, Ulrike (2009): "Kontexte für nicht-kanonische Verbzweitstellung: V2 nach dass und Verwandtes". In: Ehrich, Veronika et al. (eds.) (2009): Koordination und Subordination im Deutschen. Hamburg, Buske: 113-134.

Giacoma, Luisa/Kolb, Susanne (eds.) (2001): Großwörterbuch Italienisch: ItalienischDeutsch/Deutsch-Italienisch. Bologna: Zanichelli/Stuttgart: Klett.

Günthner, Susanne (1996): From subordination to coordination? Verb-second position in German causal and concessive constructions. Arbeitspapier Fachgruppe Sprachwissenschaft der Universität Konstanz.

Günthner, Susanne (1999a): "Entwickelt sich der Konzessivkonnektor obwohl zum Diskursmarker? Grammatikalisierungstendenzen im gesprochenen Deutsch". Linguistische Berichte 180: 409-446.

Günthner, Susanne (1999b): "Wenn-Sätze im Vor-Vorfeld. Ihre Formen und Funktionen in der gesprochenen Sprache". Deutsche Sprache 27: 209-235.

Haegeman, Liliane (2003): "Conditional Clauses: External and internal Syntax". Mind and Language 18/4: 317-339.

Haegeman, Liliane (2004): "The syntax of adverbial clauses and its consequences for topicalisation". Antwerp Papers in Linguistics 107: 61-90.

Haegeman, Liliane (2006): "Conditionals, factives and the left periphery". Lingua 16: 16511669.

Haegeman, Liliane (2012): Adverbial Clauses, Main Clause Phenomena, and the Composition of the Left Periphery. Oxford: Oxford University Press.

Haspelmath, Martin (2004): "Coordinating constructions: An overview". In: Haspelmath, Martin (ed.) (2004): Coordinating constructions. Amsterdam, Benjamins: 3-39.

Helbig, Gerhard/Buscha, Joachim (2001): Deutsche Grammatik. Ein Handbuch für den Ausländerunterricht. Berlin: Langenscheidt.

Höhle, Tilman N. (1986): "Der Begriff 'Mittelfeld'. Anmerkungen über die Theorie der topologischen Felder". In: Schöne, Albrecht (ed.) (1986): Kontroversen, alte und neue. Akten des VII. Internationalen Germanisten-Kongresses Göttingen 1985. Bd. 3: Textlinguistik contra Stilistik u. a. (ed. Walter Weiss et al.). Tübingen, Niemeyer: 329340 . 
Holler, Anke (2005): Weiterführende Relativsätze. Empirische und theoretische Aspekte. Berlin: Akademie Verlag.

Holler, Anke (2008): "German dependent clauses from a constraint-based perspective". In: Fabricius-Hansen/Ramm (eds.) (2008): 187-216.

Johannessen, Janne Bondi (1998): Coordination. New York: Oxford University Press.

Konopka, Marek (2006): "Topologie komplexer Sätze und Textverstehen. Zur Stellung von Verbletztsätzen mit weil". In: Blühdorn, Hardarik/Breindl, Eva/Waßner, Ulrich H. (eds.) (2006): Text-Verstehen. Grammatik und darüber hinaus. Berlin, de Gruyter: 108-124.

Kortmann, Bernd (1996): Adverbial Subordination. A Typology and History of Adverbial Subordinators Based on European Languages. Berlin: Mouton de Gruyter.

Lohnstein, Horst (2004): "Variable und invariante Strukturmerkmale von Satzkonnektoren". In: Blühdorn, Hardarik/Breindl, Eva/Waßner, Ulrich Hermann (eds.) (2004): Brücken schlagen. Grundlagen der Konnektorensemantik. Berlin/New York, de Gruyter: 137-160.

Lohnstein, Horst (2014): "Artenvielfalt in freier Wildbahn - Generative Grammatik". In: Hagemann, Jörg/Staffeldt, Sven (eds.) (2014): Syntaxtheorien. Analysen im Vergleich. Tübingen, Stauffenburg: 165-185.

Lombardi Vallauri, Edoardo (2000): Grammatica funzionale delle avverbiali italiane. Roma: Carocci.

Müller, Natascha/Riemer, Beate (1998): Generative Syntax der romanischen Sprachen. Französisch, Italienisch, Portugiesich, Spanisch. Tübingen: Stauffenburg.

Pasch, Renate et al. (2003): Handbuch der deutschen Konnektoren. Berlin/New York: de Gruyter.

Pittner, Karin (1999): Adverbiale im Deutschen. Untersuchungen zu ihrer Stellung und Interpretation. Tübingen: Stauffenburg.

Previtera, Luisa (1996): "I costrutti causali". Studi Italiani di Linguistica Teorica e Applicata 1/XXV: 29-46.

Ramers, Karl Heinz (2006): "Topologische Felder. Nominalphrase und Satz im Deutschen". Zeitschrift für Sprachwissenschaft 25: 95-127.

Ravetto, Miriam/Blühdorn, Hardarik (2011): "Die Kausalkonjunktionen denn, weil, da im Deutschen und perché, poiché, siccome im Italienischen". In: Ferraresi, Gisella (ed.) (2011): Konnektoren im Deutschen und im Sprachvergleich. Beschreibung und grammatische Analyse. Tübingen, Narr: 207-250.

Reich, Ingo et al. (2009): "Einführung". In: Ehrich, Veronika et al. (eds.) (2009): Koordination und Subordination im Deutschen. Hamburg: Buske: 5-20.

Reis, Marga (1997): "Syntaktischer Status unselbständiger Verbzweit-Sätze". In: Dürscheid, Christa/Ramers, Karl Heinz/Schwarz, Monika (eds.) (1997): Sprache im Fokus. Festschrift für Heinz Vater zum 65. Geburtstag. Tübingen, Niemeyer: 121-144.

Reis, Marga/Wöllstein, Angelika (2010): "Zur Grammatik (vor allem) konditionaler V1Gefüge im Deutschen". Zeitschrift für Sprachwissenschaft 29: 111-179.

Renzi, Lorenzo/Salvi, Giampaolo/Cardinaletti, Anna (2001): Grande grammatica italiana di consultazione. Bologna: Il Mulino.

Rizzi, Luigi (1997): "The fine structure of the left periphery". In: Haegeman, Liliane (ed.) (1997): Elements of Grammar. Handbook in Generative Syntax. Dordrecht/Boston/London, Kluwer: 281-337.

Schwarze, Christoph ( $\left.{ }^{2} 1995\right)$ : Grammatik der italienischen Sprache. Tübingen: Niemeyer. 
Serianni, Luca (2000): Italiano. Grammatica. Sintassi. Dubbi. Milano: Garzanti.

Sternefeld, Wolfgang (2008/2009): Syntax. Eine morphologisch motivierte generative Beschreibung des Deutschen. Tübingen: Stauffenburg.

Sweetser, Eve (1990): From etymology to pragmatics. Metaphorical and cultural aspects of semantic structure. Cambridge: Cambridge University Press.

Uhmann, Susanne (1998): "Verbstellungsvariation in weil-Sätzen". Zeitschrift für Sprachwissenschaft 17: 92-139.

Volodina, Anna (2011): Konditionalität und Kausalität im Diskurs. Eine korpuslinguistische Studie zum Einfluss von Syntax und Prosodie auf die Interpretation komplexer Äußerungen. Tübingen: Narr.

Zifonun, Gisela et al. (1997): Grammatik der deutschen Sprache. Berlin/New York: de Gruyter. 